\title{
The Appearance and Disappearance of Exocomet Gas Absorption
}

\author{
Barry Y. Welsh ${ }^{1}$ and Sharon L. Montgomery ${ }^{2}$ \\ ${ }^{1}$ Experimental Astrophysics Group, Space Sciences Laboratory, University of California, 7 Gauss Way, Berkeley, CA 94720, USA \\ ${ }^{2}$ Department of Chemistry, Mathematics \& Physics, Clarion University, Clarion, PA 16214, USA \\ Correspondence should be addressed to Barry Y. Welsh; bwelsh@ssl.berkeley.edu
}

Received 30 March 2015; Accepted 9 June 2015

Academic Editor: De-Fu Bu

Copyright (C) 2015 B. Y. Welsh and S. L. Montgomery. This is an open access article distributed under the Creative Commons Attribution License, which permits unrestricted use, distribution, and reproduction in any medium, provided the original work is properly cited.

CaII K-line (3933 $\AA$ ) absorption profiles observed towards 15 A-type and two B-type stars with known (or suspected) debris disks, in addition to archival spectral data for three A-type stars, are presented. Inspection of the extracted CaII K-line absorption lines has determined that one late B-type (HD 58647) and four new A-type (HD 56537, HD 64145, HD 108767, and HD 109573) stellar systems exhibit short-term (night-to-night) absorption variation within these profiles. This variability is due to the liberation of gas from falling evaporating bodies (the so-called FEBs activity) on the grazing approach of exocomets towards their parent star. Also we present archival spectra of the FeI $\lambda 3860 \AA$ and CaII K-lines towards $\beta$ Pictoris (HD 39060) that, for the first time, reveal the absorption signatures of FEB activity at similar velocities in both line profiles. FEB-hosting stars seem to be of an earlier type (on average spectral type Al.8 as compared with A3.1) than those where no FEB activity has been observed. This could be due to stellar activity levels (such as chromospheric activity or nonradial pulsations) being of a higher level within the atmospheres of these hotter A-type stars.

\section{Introduction}

The existence of reservoirs of small rocky bodies (i.e., asteroids/planetesimals) in orbits around many stellar systems is now well established. Their presence is required by current planetary formation theories $[1,2]$ and also through direct and indirect observations of stars with associated dusty and gaseous circumstellar disks [3-5]. Their appearance in large numbers close to the host star is likely episodic and triggered by the formation of large planets [6]. Since dust and gasrich systems will transition to optically thin and gas-free debris disks, observation of asteroids/planetesimals is best performed in young or debris-rich systems, which may have associated planetary bodies in the early stages of formation. In such systems, the gravitation fields can potentially enable the disruption of large numbers of these kilometer-sized icy bodies into trajectories directed towards the young central star. Indeed the detection of evaporating gas liberated from the comae of swarms of these infalling comet-like bodies (or exocomets) can be interpreted as being a signature of the early stages of the formation of a planetary system. To date, the search for exocomets has focused on young
( $<50 \mathrm{Myr}$ ) A-type stars, since roughly one-third of normal Atype main sequence stars exhibit infrared (IR) excesses due to the presence of dusty debris disks $[7,8]$. Since the radiation pressure from A-type stars removes gas and dust particles from the disk on timescales $\ll 1 \mathrm{Myr}$ [9], then any dust and/or gas found close to the star must have arisen recently from the collision and evaporation events amongst planetesimals.

The evaporation of gaseous products from infalling exocomets occurring close to a star can potentially cause small disruptions in the ambient circumstellar disk plasma. For circumstellar disks that are viewed "edge-on" this evaporating material may be directly observed through transient gas absorption features seen at rapidly changing redshifts. This short-term (night-to-night) variability in absorption was first detected towards the $\mathrm{A} 6 \mathrm{~V}$ star $\beta$ Pictoris, through repeated observations of the CaII K-line at $3933 \AA[10,11]$. The relatively warm $(T \sim 100 \mathrm{~K}-2500 \mathrm{~K})$ gas liberated during these evaporative events generally lies within $\sim 1 \mathrm{AU}$ of the star, whereas the cold and optically thick circumstellar gas disk (as traced by CO emission) is located at further distances $>20$ AU from the central star [12]. 
Over the last few years renewed interest in the detection of exocomet activity through observation of short-term (night-to-night) absorption variability of the circumstellar CaII $3933 \AA$ K-line towards A-type stars has resulted in the addition of over a dozen new stellar systems thought to be somewhat similar to that of $\beta$ Pictoris $[3,14,15]$. The release of evaporating gas from these kilometer-sized "falling evaporating bodies" (FEBs or exocomets) generally requires their orbits to be perturbed by an outer planet onto stargrazing orbits [16]. For the case of $\beta$ Pictoris this prompted a twenty-year search for an associated exoplanet, which culminated in the detection of the giant planet, $\beta$ Pictoris $b$, by Lagrange et al. [17]. The widely used techniques of exoplanet detection towards (older) stars of late spectral types $G$ to $M$, via radial velocity variation or the observation of transits, are generally ill-suited to young A-type stars. Hence, the detection of short-term exocomet activity in such young ( $<50 \mathrm{Myr}$ old) systems may currently be the best indication of the presence of associated exoplanets, whose existence can then be confirmed using very high contrast direct imaging techniques. This is of particular importance since recent high contrast imaging observations suggest that fewer than $10 \%$ of B- and A-type stars may possess giant exoplanets (of mass > $10 \mathrm{M}_{\mathrm{JUP}}$ ) at distances between 40 and $650 \mathrm{AU}$ [18].

An analysis of $\sim 30$ rapidly rotating A-type stars that have been repeatedly observed in absorption at the CaII K-line suggests that those (ten) stars that exhibit FEB activity are younger $(<100 \mathrm{Myr})$ and are more likely to exhibit chemical peculiarities than those A-type stars in which FEB activity has not been detected [3]. Interestingly, FEB activity does not appear to be directly associated with a strong mid-IR excess. This is most probably linked to the viewing angle of the circumstellar gas disk. Even if a dust disk or infalling comets are present, they will be very difficult to detect if the viewing angle is not within a few degrees of edge-on [16]. We note that $\beta$ Pictoris, the system in which FEB activity is most frequently observed, has a disk with an inclination angle of $88.5^{\circ}$ [29]. In addition, the orbital inclination of the inflating comets is also a prime factor in determining the observed velocity of an FEB event, and for the case of $\beta$ Pictoris the angle between the axis of the cometary orbit and the line of sight is tightly constrained to be within $10^{\circ}$ of $150^{\circ}$ [16]. In the absence of direct imaging, the only way we have of selecting near edge-on disk systems is by observing stars that rapidly rotate $\left(V \sin i>120 \mathrm{~km} \mathrm{~s}^{-1}\right)$. Given that $V \sin i$, an imperfect proxy for viewing angle, it is perhaps not surprising that the (10) stars in which FEB activity has thus far been detected are not characterized by significantly higher rotation velocities when compared with those (21) stars in which FEB activity was not detected by Welsh and Montgomery [3].

$\beta$ Pictoris, an A6V star, has subsequently become the de facto canonical young ( $12 \mathrm{Myr})$ A-type star with an IR excess and a debris disk containing planetesimal belts containing myriads of small rocky bodies $[6,30]$. In addition to showing absorption variability at the circumstellar CaII K-line on a night-to-night (and sometimes hourly) basis, $\beta$ Pictoris also exhibits absorption from the metastable $3 \mathrm{~d}$ levels of the CaII IR triplet at $8542 \AA[31,32]$. Such absorption, which is due to radiative pumping, can only occur within a circumstellar environment and cannotbe of interstellar origin. These observations indicate the location of the warm circumstellar gas around the central star to be $<10 \mathrm{AU}$. Such infalling gas would appear to possess a high electron density $\left(n_{e}>10^{6} \mathrm{~cm}^{-3}\right)$ and also a high electron temperature $\left(T_{e}>15,000 \mathrm{~K}\right)$. $\beta$ Pictoris also exhibits two other types of circumstellar absorption behavior at visible wavelengths. Firstly, the main circumstellar CaII K-line component arises close to (or at) the stellar radial velocity (i.e., $V_{\text {helio }} \sim+22.0 \mathrm{~km} \mathrm{~s}^{-1}$ ) which is located at a slight red shift of $\sim+2 \mathrm{~km} \mathrm{~s}^{-1}$ from that of the far weaker circumstellar NaI D-line absorption [33]. Fernández et al. [34] have explained this velocity difference by a gas braking mechanism in which neutral circumstellar species are rapidly ionized by the strong stellar radiation force and thus only reach limited (and slightly different) velocities.

Typical column density ratios of $\mathrm{N}(\mathrm{CaII}) / \mathrm{N}(\mathrm{NaI})>30: 1$ have been determined for the gas at the central velocity of the CaII K-line. These values are at least an order of magnitude higher than those found for the general interstellar medium. A second phenomenon that seems peculiar only to the $\beta$ Pictoris system is the unusually high level of circumstellar absorption associated with the rarely observed FeI $3860 \AA$ line. This spectral feature is generally found to be very weak in the interstellar medium, being found mainly in cold and dense regions [35]. However, for the case of $\beta$ Pictoris, it has an equivalent width of $\sim 6 \mathrm{~m} \AA$ with the circumstellar absorption being centered near the radial velocity of the star (see Section 3.1.8 of this paper).

Now since FEB activity has been detected towards several other nearly A-type stars, it is instructive to pose the question as to whether $\beta$ Pictoris really is a typical example of an A-type stellar system with a debris disk that exhibits FEB activity due to the evaporation of exocomets. We note that, of 16 debris discs around A-type stars that have been resolved by imaging in the IR, eleven show evidence of multiple dust components [36]. These dust disks exhibit a wide diversity in physical appearance, with radii from a few AU to hundreds of AU. Some of these disks are confined dust rings with clear inner and outer edges as found around HR 4796 by Schneider et al. [37], whereas some have very extended disks like that of $\beta$ Pictoris [38]. Additionally, several authors have posited that the origin of inner dust (and gas) disks around A-type stars might be associated with a stellar phenomenon, even though their mass-loss rates are generally very low and should not give rise to significant near-infrared emission. However, we note that recent work has shown a possible correlation between the K-band IR excess and the rotational velocity of such stars, which would hint at a possible stellar mass-loss origin for the circumstellar dust (and gas) [39].

Other spectral types of young A-type stars have also been found to exhibit temporal absorption variability of the CaII K-line that can be associated with a surrounding circumstellar disk. These are the young (but metal poor) $\lambda$ Bootis-type stars that are surrounded by a thick cloud of dusty gas $[40,41]$. In addition, the premain sequence Herbig $\mathrm{Ae} / \mathrm{Be}$ stars typically also have large IR excesses with associated circumstellar gas/dust disks [42-44]. Since it is widely 
TABLE 1: Stellar target information.

\begin{tabular}{|c|c|c|c|c|c|c|c|c|}
\hline Name & R.A. (2000) & Dec. (2000) & $m v$ & $\mathrm{Sp}$ & $\begin{array}{c}\text { Distance } \\
(\mathrm{pc})\end{array}$ & $\begin{array}{c}V \sin i \\
\left(\mathrm{~km} \mathrm{~s}^{-1}\right)\end{array}$ & $\begin{array}{l}\text { Radial velocity } \\
\left(\mathrm{km} \mathrm{s}^{-1}\right)\end{array}$ & $\begin{array}{l}\text { Age } \\
\text { Myr }\end{array}$ \\
\hline HD 31295 & $04: 54: 54$ & $+10: 09: 03$ & 4.7 & A3V & 36 & 120 & +11.1 & $100^{\mathrm{d}, \mathrm{e}}$ \\
\hline HD 38678 & $05: 46: 57$ & $-14: 49: 10$ & 3.5 & A2IV & 22 & 259 & +24.7 & $170^{\mathrm{d}, \mathrm{e}, \mathrm{f}}$ \\
\hline HD 64145 & $07: 53: 30$ & $+26: 45: 57$ & 5.0 & $\mathrm{~A} 3 \mathrm{~V}$ & 68 & 165 & +8.0 & Unknown \\
\hline HD 184006 & $19: 29: 42$ & $+51: 43: 47$ & 3.8 & A5V & 37 & 240 & $-19.5 \pm 2.0$ & $450^{g}$ \\
\hline HD 56537 & 07:18:06 & $+16: 32: 25$ & 3.6 & A3V & 31 & 154 & $-7.4 \pm 1$ & $550^{g}$ \\
\hline HD 58647 & $07: 25: 56$ & $-14: 10: 44$ & 6.9 & B9IVe & 320 & 118 & tbd & $0.4^{\mathrm{e}}$ \\
\hline HD 71155 & $08: 25: 40$ & $-03: 54: 23$ & 3.9 & $\mathrm{AOV}$ & 38 & 134 & $+10.0 \pm 1.8$ & $135^{\mathrm{d}, \mathrm{g}}$ \\
\hline HD 88195 & 10:10:08 & $-08: 24: 29$ & 5.9 & AOVsh & 183 & 213 & $+21.0 \pm 1.1$ & $0.3^{\mathrm{e}}$ \\
\hline HD 95418 & 11:01:50 & $+56: 22: 57$ & 2.4 & $\mathrm{AlV}$ & 24 & 46 & $-13.1 \pm 0.1$ & $405^{\mathrm{d}, \mathrm{g}}$ \\
\hline HD 106591 & $12: 15: 26$ & $+57: 01: 57$ & 3.3 & $\mathrm{~A} 3 \mathrm{~V}$ & 25 & 233 & $-15.3 \pm 0.5$ & $490^{\mathrm{g}}$ \\
\hline HD 108767 & $12: 29: 52$ & $-16: 30: 56$ & 2.9 & AOlV & 27 & 236 & $+13.9 \pm 2.3$ & $260^{\mathrm{g}}$ \\
\hline HD 112429 & $12: 55: 29$ & $+65: 26: 19$ & 5.2 & A5V & 29 & 120 & $+9.0 \pm 2$ & $50^{\mathrm{a}}$ \\
\hline HD 125161 & $14: 16: 10$ & $+51: 22: 02$ & 4.8 & A7V & 29 & 144 & $-18.7 \pm 2.0$ & $40^{\mathrm{g}}$ \\
\hline HD 158352 & $17: 28: 50$ & $+00: 19: 50$ & 5.4 & A7V & 60 & 180 & $-36.1 \pm 2.0$ & $600^{\mathrm{d}}$ \\
\hline HD 9672 & 01:34:37 & $-15: 40: 34.9$ & 5.6 & $\mathrm{AlV}$ & 61 & 196 & $+10.3 \pm 1.1$ & $40^{\mathrm{h}}$ \\
\hline HD 80007 & 09:13:12 & $-69: 43: 02$ & 1.7 & AlIII & 35 & 145 & $-5.1 \pm 2.3$ & $260^{\mathrm{b}}$ \\
\hline HD 135742 & $15: 17: 00$ & $-09: 22: 58$ & 2.6 & $\mathrm{~B} 8 \mathrm{~V}$ & 57 & 250 & $-35.6 \pm 1.8$ & $100^{c}$ \\
\hline
\end{tabular}

${ }^{\mathrm{a}}$ Chen et al. [19], ${ }^{\mathrm{b}}$ Rieke et al. [20], ${ }^{\mathrm{c}} \mathrm{Su}$ et al. [7], ${ }^{\mathrm{d}}$ Rhee et al. [21], ${ }^{\mathrm{e}}$ Montesinos et al. [22], ${ }^{\mathrm{f}}$ Rodriguez and Zuckerman [23], ${ }^{\mathrm{g}}$ Vican [24], and ${ }^{\mathrm{h}}$ Zuckerman and Song [5]. Stellar radial velocities from Gontcharov [25].

accepted that circumstellar disks are a common feature of both star formation and planetary formation processes, then comparing the variability behavior of circumstellar disk gas surrounding all types of young A-type stars of differing ages (1-100 Myr) may provide insights into the early stages of the evolution of planetary bodies.

In this paper we present nightly spectral observations of 15 A-type stars (plus two late B-type stars) with known (or suspected) debris disks, in addition to presenting similar spectral data for three A-type stars retrieved from the European Southern Observatory data archive. In Section 2 we describe how we have derived the residual intensity absorption profiles of the circumstellar CaII K-line from each observation to measure the corresponding line equivalent width value. These measurements are discussed in Section 3 for each target star, and we have determined that one late B-type (HD 58647) and four new A-type (HD 56537, HD 64145, HD 108767, and HD 109573) stars exhibit short-term (night-to-night) variation in the equivalent width values of their circumstellar CaII K-line absorption line profiles. Based on the similar behavior of CaII absorption exhibited by the $\beta$ Pictoris stellar system, we associate this absorption variability with the evaporation of gas from comet-like bodies on their grazing approach to a parent star and note that this brings the total number of systems thought to harbor exocomets to 16 (see Table 4), 13 of which have been discovered by the present authors.

\section{Observations and Data Extraction}

High spectral resolution observations of a total of 17 stars listed in Table 1 are presented in this paper. With the exception of the radial velocities [25], the astrophysical data for each star shown in Table 1 have been drawn from the SIMBAD data retrieval system. Target selection was based on the physical properties shared with other circumstellar gas systems already known to exhibit variable absorption at the CaII wavelengths. Thus, most of the program targets are similarly young, nearby $(d<100 \mathrm{pc})$ A-type main sequence stars that rotate rapidly and exhibit a mid-infrared excess. Such A-type stars often possess a known (or suspected) associated debris disk $[26,45]$, which may have the potential to harbor exocomet activity.

Spectral observations of the four stars listed in the uppermost section of Table 1 were performed in the service mode by staff on the $2.1 \mathrm{~m}$ Bernard Lyot telescope at the Pic du Midi Observatory in France. Spectra for three stars were recorded on four nights over the time period 10-28- to 111-2014, and the star HD 184006 was similarly observed on three occasions over the time period 09-21- to 10-7-2013. The intent of these (and all of our) observations was to observe each of the targets (weather permitting) on a nightly basis to search for absorption variability of the CaII K-line at $3933 \AA$. Each observation consisted typically of an integration time of $\sim 60$ minutes, such that the spectra were well exposed with typical $S / N$ ratios of $\sim 200: 1$ in the stellar continuum around the CaII K-line. Medium spectral resolution observations of $R \sim 65,000\left(4.6 \mathrm{~km} \mathrm{~s}^{-1}\right)$ were obtained for the 3 stars over the entire 3705-9485 $\AA$ range using the EsPaDOns echelle spectrograph used in the "star only" mode. Spectra were recorded with a EEV CCD of $2000 \times 4500$ pixels and the data were processed at the Observatory using the Libre ESpRIT data reduction software tool, which uses many of the same data extraction techniques outlined in the last paragraph of this section. 
TABLE 2: ESO data archival observations.

\begin{tabular}{|c|c|c|c|c|c|c|c|c|}
\hline Name & $m v$ & $\mathrm{Sp}$ & $\begin{array}{c}\text { Distance } \\
(\mathrm{pc})\end{array}$ & $\begin{array}{c}V \sin i \\
\left(\mathrm{~km} \mathrm{~s}^{-1}\right)\end{array}$ & $\begin{array}{c}\text { Radial velocity } \\
\left(\mathrm{km} \mathrm{s}^{-1}\right)\end{array}$ & $\begin{array}{l}\text { Age } \\
\text { Myr }\end{array}$ & Instrument & Observation date \\
\hline \multirow{4}{*}{ HD 109573 (HR 4796) } & \multirow{4}{*}{5.8} & \multirow{4}{*}{$\mathrm{AO}$} & \multirow{4}{*}{73} & \multirow{4}{*}{152} & \multirow{4}{*}{$7.1 \pm 1.1$} & \multirow{4}{*}{$8^{\mathrm{a}}$} & FEROS & 2007-03-09Т06:23:49 \\
\hline & & & & & & & FEROS & 2007-05-04T02:27:33 \\
\hline & & & & & & & FEROS & 2007-05-04T02:43:21 \\
\hline & & & & & & & FEROS & 2010-05-27Т00:28:30 \\
\hline \multirow{5}{*}{ HD 161868} & \multirow{5}{*}{3.8} & \multirow{5}{*}{$\mathrm{AOV}$} & \multirow{5}{*}{32} & \multirow{5}{*}{210} & \multirow{5}{*}{$-7.6 \pm 0.3$} & \multirow{5}{*}{$184^{\mathrm{a}}$} & FEROS & 2005-08-19T23:23:43 \\
\hline & & & & & & & FEROS & 2005-08-20T00:00:46 \\
\hline & & & & & & & FEROS & 2005-09-09T00:10:46 \\
\hline & & & & & & & FEROS & 2006-05-20T05:42:34 \\
\hline & & & & & & & FEROS & 2006-09-12T01:43:21 \\
\hline \multirow{3}{*}{ Beta Pictoris (HD 39060) } & \multirow{3}{*}{3.9} & \multirow{3}{*}{$\mathrm{A} 6 \mathrm{~V}$} & \multirow{3}{*}{19} & \multirow{3}{*}{122} & \multirow{3}{*}{$20.0 \pm 0.7$} & \multirow{3}{*}{$12^{\mathrm{b}}$} & HARPS & 2003-12-23Т00:35:22 \\
\hline & & & & & & & HARPS & 2003-12-28T00:17:03 \\
\hline & & & & & & & HARPS & 2003-12-29T08:43:28 \\
\hline
\end{tabular}

${ }^{\mathrm{a}}$ Chen et al. [26], and ${ }^{\mathrm{b}}$ Rhee et al. [21].

In the second section of Table 1 we present information regarding repeated spectroscopic observations of $11 \mathrm{~A}$ - or Btype stars using the Sandiford Echelle Spectrograph mounted at the Cassegrain focus of the $2.1 \mathrm{~m}$ telescope at the McDonald Observatory, Texas. Medium spectral resolution observations of $R 60,000\left(5 \mathrm{~km} \mathrm{~s}^{-1}\right)$ were obtained over the $3800-5000 \AA$ region and the data was recorded with a Reticon CCD detector of $1200 \times 400$ pixels. The first nine of these stars were observed over the nights of 03-1- to 03-7-2013. The tenth star, HD 158352, was observed in an identical fashion on four occasions over the time period 08-18- to 08-23-2010. We also present CaII K-line absorption observations of the star HD 9672 (49 Ceti) recorded on the nights of 08-11-12-2013 using the same instrumental set-up. This star was previously known to exhibit FEB absorption activity similar to that of $\beta$ Pictoris [15] and these observations were chosen to simultaneously coincide with ultraviolet absorption observations recorded with the STIS instrument aboard the Hubble Space Telescope. Preliminary results from observations of the neutral ultraviolet absorption species detected towards 49 Ceti have been presented by Roberge et al. [46].

Finally spectral observations of the two stars listed in the lowest section of Table 1 were performed during the nights of 05-8- to 05-13-2014 using the GIRAFFE fiber-fed echelle spectrograph mounted on the $1.9 \mathrm{~m}$ Radcliffe telescope of the South African Astronomical Observatory. The spectral data which encompassed a wavelength range of $3850 \AA-5500 \AA$ were recorded with a Tektronix $1024 \times 1024$ CCD detector at a resolving power of $\sim 40,000\left(7.5 \mathrm{~km} \mathrm{~s}^{-1}\right)$.

All of the spectral data recorded at the various observatories were extracted and reduced in a similar manner. The recorded images were bias-subtracted and flat-fielded using standard IRAF echelle data reduction routines. Cosmic rays and bad pixels were removed, and the spectral orders were then extracted using the standard optimal spectrum extraction algorithm of Horne [47]. The wavelength dispersion calibration of the stellar spectra was obtained by cross-reference to Th-Ar emission spectra recorded at the beginning and end of each night. This resulted in a wavelength accuracy of $\sim 0.015 \AA\left(1 \mathrm{~km} \mathrm{~s}^{-1}\right)$ for all of the stellar spectra. These wavelengths were then transformed into the heliocentric frame of reference for all future discussion in this paper.

2.1. Archival Data. We have also made a cursory inspection of some of the numerous observations of A-type stars recorded with the FEROS and/or HARPS high-resolution spectrographs that are deposited within the European Southern Observatory (ESO) data archive (http://archive.eso.org/cms .html). In Table 2 we list three A-type stars (together with their astrophysical information) for which we have downloaded spectral data that have been processed using the standard ESO data pipeline. We list the dates of each of these observations together with the spectrograph that recorded these spectra. We note that the HARPS spectral data are recorded with a resolving power of $R \sim 110,000$, whereas the FEROS spectral data have a lower resolving power of $R \sim$ 48,000 . In addition to the $\mathrm{CaII}$ and $\mathrm{NaI}$ absorption lines, the FeI $3860 \AA$ circumstellar absorption line was also extracted from the $\beta$ Pictoris spectra. None of the other targets listed in Tables 1 and 2 had any significant absorption feature in their spectra near this wavelength to a $3 \sigma$ upper limit of EW $<1.5 \mathrm{~m} \AA$.

2.2. Absorption Line Analysis. All of the observed spectra contained a rotationally broadened stellar (CaII-K) absorption line centered at $3933 \AA$, most of which also had a very narrow circumstellar absorption line superposed at the bottom of the stellar absorption trough. A typical example of this type of absorption profile can be seen in Figure 1 of Montgomery and Welsh [15]. This sort of spectral signature is typical of A-type stars that possess circumstellar gaseous debris disks corotating near to their stellar radial velocity $[3,15,27,28]$.

A stellar continuum level for each target was determined by fitting the broad CaII K-line photospheric absorption 



FIgURE 1: The circumstellar CaII K-line absorption spectra of HD 64145 recorded on 4 nights in October 2014. Note the appearance of an extra absorption feature centered at $V \sim-15 \mathrm{~km} \mathrm{~s}^{-1}$ on the night of 10-31-2014, which is probably an FEB event.

trough with a 6th (or higher) order polynomial over a velocity range of $\pm 150 \mathrm{~km} \mathrm{~s}^{-1}$ from the central absorption. This resulted in a residual intensity absorption profile for the far weaker circumstellar CaII K-line. The absorption equivalent width of this circumstellar line was then determined from a straightforward integration of the residual intensity spectrum summed over the line profile. The continuum placement software assigns an rms error to each of the spectral data points, which is subsequently adopted as the $1-\sigma$ error for these points as defined in Vallerga et al. [48].

All of the spectra of our target stars may have some (small) contribution due to interstellar absorption along their sightline path lengths. Hence, in Table 3 we also list the projected velocity vector of the local interstellar cloud (LIC) for each sightline using the respective cloud properties listed in Table 16 of Redfield and Linsky [13]. In many cases these velocities can be used to discriminate between circumstellar absorption features (which should form at velocities close to the radial velocity of the star) and any local interstellar features.

\section{Discussion of Individual Target Spectra}

Previous observations of several local A-type stars with debris disks have shown two main types of circumstellar absorption variability. First, the absorption strength or shape of the absorption profile of the main circumstellar CaII K-line (usually centered at the radial velocity of the star) may change over time periods on the order of days (or in some cases on an hourly basis). Secondly, transient weak absorption features (FEBs) may occasionally appear at velocities that are red or blue-shifted by tens of $\mathrm{km} \mathrm{s}^{-1}$ away from the main circumstellar K-line, which itself may often vary in absorption strength. An assessment of a significant change in the main circumstellar line can be obtained by comparison of 
TABLE 3: Stars showing no change in CaII K-line absorption.

\begin{tabular}{|c|c|c|c|c|c|c|}
\hline Star & Dates observed & $\begin{array}{c}\text { CaII-K (EW)av } \\
\mathrm{m} \AA \\
\end{array}$ & $\begin{array}{c}\text { Line velocity } \\
\left(\mathrm{km} \mathrm{s}^{-1}\right)\end{array}$ & $\begin{array}{c}\text { LISM velocity } \\
\left(\mathrm{km} \mathrm{s}^{-1}\right)\end{array}$ & $\begin{array}{c}\text { Radial velocity } \\
\left(\mathrm{km} \mathrm{s}^{-1}\right)\end{array}$ & ISM or $\mathrm{C} / \mathrm{S}^{*}$ \\
\hline HD 88195 & 03-01- to $03-07-2013$ & $42.8 \pm 4.0$ & $+9.0,+21.8$ & $+9.2,+20.5$ & +21.0 & ISM \\
\hline HD 106591 & 03-01- to $03-07-2013$ & $5.5 \pm 1.0$ & $-1.0,+12.5,+25.0$ & +2.1 & -15.3 & ISM \\
\hline HD 135742 & 05-08- to $05-13-2014$ & $7.5 \pm 1.0$ & -24.0 & -27.0 & -35.6 & ISM \\
\hline HD 158352 & $08-18$ - to $08-23-2010$ & $25.5 \pm 2.0$ & $-37.0,-28.0$ & -28.1 & -36.1 & $\mathrm{C} / \mathrm{S}+\mathrm{ISM}$ \\
\hline HD 161868 & August 2005 to September 2006 & $7.3 \pm 0.8$ & -29.0 & -27.3 & -7.6 & ISM \\
\hline HD 184006 & 09-21- to $10-07-2013$ & $14.5 \pm 2.0$ & $-8.3,-17.4$ & -7.8 & -19.5 & $\mathrm{C} / \mathrm{S}+\mathrm{ISM}$ \\
\hline
\end{tabular}

${ }^{*}$ The observed CaII absorption line is mainly of interstellar (ISM) or circumstellar (C/S) origin.

TABle 4: Physical parameters of A-stars with circumstellar CaII K-line absorption.

\begin{tabular}{|c|c|c|c|c|c|c|c|c|}
\hline Star HD\# & Spectral type & $\begin{array}{c}V \sin i \\
\left(\mathrm{~km} \mathrm{~s}^{-1}\right)\end{array}$ & $\begin{array}{c}\text { Age } \\
\text { (Myr) }\end{array}$ & Mid-IR excess? & Chemically peculiar? & $\lambda$ Boo star? & $\begin{array}{c}\text { EW } \lambda 4481 \\
(A)\end{array}$ & Reference \\
\hline \multicolumn{9}{|c|}{ Stars not showing FEB activity } \\
\hline HD 21688 & A5III/IV shell & 196 & Unknown & $\mathrm{Y}$ & Unlisted & $\mathrm{N}$ & 0.52 & {$[3]$} \\
\hline HD 38090 & A2/A3V shell & 207 & Unknown & Unknown & $\mathrm{N}$ & $\mathrm{N}$ & 0.47 & {$[3]$} \\
\hline HD 38545 & A3V shell & 191 & 13 & $\mathrm{~N}$ & $\mathrm{~N}$ & $\mathrm{~N}$ & 0.42 & {$[3]$} \\
\hline HD 39182 & A2V shell & 238 & Unknown & Unknown & $\mathrm{N}$ & $\mathrm{N}$ & 0.38 & {$[27]$} \\
\hline HD 80007 & $\mathrm{~A} 2 \mathrm{IV} / \mathrm{V}$ & 145 & 260 & $\mathrm{~N}$ & Unlisted & $\mathrm{N}$ & Unlisted & This work \\
\hline HD 102647 & $\mathrm{~A} 3 \mathrm{~V}$ & 110 & 50 & $\mathrm{Y}$ & $\mathrm{N}$ & $\mathrm{N}$ & 0.57 & {$[3]$} \\
\hline HD 141569 & AOVe & 228 & 5.4 & $\mathrm{Y}$ & Unlisted & $\mathrm{N}$ & Unlisted & {$[3]$} \\
\hline HD 148283 & $\mathrm{~A} 5 \mathrm{~V}$ & 280 & Unknown & $\mathrm{N}$ & $\mathrm{N}$ & $\mathrm{N}$ & 0.4 & {$[3]$} \\
\hline HD 158352 & A7V shell & 180 & 600 & $\mathrm{Y}$ & $\mathrm{N}$ & $\mathrm{N}$ & 0.60 & This work \\
\hline HD 165459 & $\mathrm{AlV}$ & Unknown & 5 & $\mathrm{Y}$ & Unlisted & $\mathrm{N}$ & Unlisted & {$[3]$} \\
\hline HD 183324 & $\mathrm{AOV}$ & 110 & 10 & $\mathrm{Y}$ & $\mathrm{Y}$ & $\mathrm{Y}$ & 0.16 & {$[3]$} \\
\hline HD 184006 & $\mathrm{~A} 5 \mathrm{~V}$ & 210 & 450 & $\mathrm{~N}$ & $\mathrm{~N}$ & $\mathrm{~N}$ & 0.50 & This work \\
\hline HD 223884 & A5V shell & 241 & 5.5 & $\mathrm{~N}$ & $\mathrm{~N}$ & $\mathrm{~N}$ & 0.46 & {$[27]$} \\
\hline \multicolumn{9}{|c|}{ Stars exhibiting FEB activity } \\
\hline HD 256 & A2IV/V shell & 241 & 1.8 & $\mathrm{~N}$ & $\mathrm{~N}$ & $\mathrm{~N}$ & 0.31 & {$[28]$} \\
\hline HD 9672 & AlV & 196 & 8.9 & $\mathrm{Y}$ & $\mathrm{N}$ & $\mathrm{N}$ & 0.64 & {$[15]$} \\
\hline HD 21620 & AOVn shell & 217 & 80 & $\mathrm{Y}$ & $\mathrm{Y}$ & $\mathrm{N}$ & 0.33 & {$[3]$} \\
\hline HD 39060 & A6V shell & 122 & 12 & $\mathrm{Y}$ & $\mathrm{N}$ & $\mathrm{N}$ & Unlisted & {$[11]$} \\
\hline HD 42111 & A3Vn shell & 120 & 0.2 & $\mathrm{~N}$ & $\mathrm{~N}$ & $\mathrm{~N}$ & 0.46 & [3] \\
\hline HD 56537 & $\mathrm{~A} 3 \mathrm{~V}$ & 154 & 550 & $\mathrm{Y}$ & $\mathrm{N}$ & $\mathrm{N}$ & 0.59 & This work \\
\hline HD 58647 & B91Ve & 118 & 0.4 & $\mathrm{Y}$ & Unlisted & $\mathrm{N}$ & Unlisted & This work \\
\hline HD 64145 & $\mathrm{~A} 3 \mathrm{~V}$ & 165 & Unknown & $\mathrm{N}$ & Unlisted & $\mathrm{N}$ & 0.64 & This work \\
\hline HD 85905 & A2/A3III shell & 264 & Unknown & $\mathrm{N}$ & Unlisted & $\mathrm{N}$ & 0.37 & {$[27]$} \\
\hline HD 108767 & AOIV & 236 & 260 & $\mathrm{Y}$ & $\mathrm{N}$ & $\mathrm{N}$ & Unlisted & This work \\
\hline HD 109573 & $\mathrm{AO}$ & 152 & 8 & $\mathrm{Y}$ & $\mathrm{N}$ & $\mathrm{N}$ & Unlisted & This work \\
\hline HD 110411 & $\mathrm{AOV}$ & 154 & 100 & $\mathrm{Y}$ & $\mathrm{Y}$ & $\mathrm{Y}$ & 0.21 & {$[3]$} \\
\hline HD 145964 & B9V & 306 & 5 & $\mathrm{~N}$ & $\mathrm{Y}$ & Weak & 0.32 & {$[3]$} \\
\hline HD 172555 & A7V & 116 & 12 & $\mathrm{Y}$ & Unlisted & $\mathrm{N}$ & Unlisted & {$[14]$} \\
\hline HD 182919 & $\mathrm{AOV}$ & 154 & 100 & $\mathrm{Y}$ & $\mathrm{N}$ & $\mathrm{N}$ & 0.44 & {$[15]$} \\
\hline HD 217782 & A3Vn shell & 212 & Unknown & $\mathrm{N}$ & $\mathrm{N}$ & Weak & 0.48 & {$[15]$} \\
\hline
\end{tabular}

its night-to-night equivalent width (EW) values, as integrated over the same velocity interval.

In the following sections we discuss the types of CaII absorption variability observed towards the 20 stars listed in Tables 1 and 2.

\subsection{Stars Exhibiting CaII Circumstellar Variability or the Presence of FEBs}

3.1.1. HD 64145. HD 64145 ( $\lambda \mathrm{Gem}$ ) is an A3V type star (of, as yet, undetermined age) located at a distance of $68 \mathrm{pc}$ with 
a warm $(T>150 \mathrm{~K})$ debris disk located close to the central star that exhibits a small IR excess at $18 \mu \mathrm{m}$ [49]. In Figure 1 we see that each of the 4 nights' observations of this star has revealed an absorption component at $V \sim$ $+9.5 \mathrm{~km} \mathrm{~s}^{-1}$, which is close to that of the stellar radial velocity. We note that the projected velocity of the local interstellar cloud according to Redfield and Linsky [13] in this galactic direction is $+18.5 \mathrm{~km} \mathrm{~s}^{-1}$, and thus we identify the nightly absorption at $V \sim+9.5 \mathrm{~km} \mathrm{~s}^{-1}$ as being of circumstellar origin. The measured EW of this line was $\sim 3.3 \mathrm{~m} \AA$ on three of the four observations, whereas the observation of 10-292014 shows the absorption strength to increase to EW = $4.6 \mathrm{~m} \AA$ which (on inspection of the associated measurement errors) is $3-\sigma$ change in value. On the night of 10-31-2014 an extra absorption feature appeared at $V \sim-10 \mathrm{~km} \mathrm{~s}^{-1}$ with an $\mathrm{EW}=2.0 \pm 0.3 \mathrm{~m} \AA$ and on night 10-30-2014 a weaker absorption feature at $V \sim-45 \mathrm{~km} \mathrm{~s}^{-1}$ is present with an EW $=1.1 \pm 0.3 \mathrm{~m} \AA$. We identify the extra absorption on $10-31-$ 2014 as being due to an FEB event due to the evaporation of exocomet gas, and although the extra absorption seen on 10$30-2014$ is only just above the continuum noise level (and is of lower statistical significance), this may also be of an FEB origin.

For comparison purposes, the main circumstellar CaII K-line detected at $V \sim+20 \mathrm{~km} \mathrm{~s}^{-1}$ towards $\beta$ Pictoris typically has an EW > $100 \mathrm{~m} \AA$. In addition, its FEB absorption components appear on a regular (< nightly) basis at velocities up to $+150 \mathrm{~km} \mathrm{~s}^{-1}$ that have an EW $>20 \mathrm{~m} \AA$ [50]. See Section 3.1.8 for a more detailed discussion of exocomet activity in the canonical $\beta$ Pictoris system.

3.1.2. HD 56537. This A3V star is located at a distance of $31 \mathrm{pc}$ and has a suspected debris disk based on its IR excess flux at $24 \mu \mathrm{m}[20,51]$. It has one of the largest $24 \mu \mathrm{m}$ IR excesses amongst older A-type stars, which has been associated with emission from an extended circumstellar (disk) structure $[20,39]$. It has an estimated age of $\sim 550 \mathrm{Myr}$ and is the oldest disk studied in our present sample of stars. Thus, we might expect that the rate of occurrence of any detectable exocomet activity would be very low. However, in Figure 2 we show that absorption variability was (unexpectedly) detected over the velocity range -20 to $+15 \mathrm{~km} \mathrm{~s}^{-1}$ on two of the six nights of observation. This velocity range encompasses that of the radial velocity of the $\operatorname{star}\left(V \sim-7.4 \mathrm{~km} \mathrm{~s}^{-1}\right)$, thus suggesting that at least some of this absorption profile is of a circumstellar origin. Figure 2 shows four of the five nights' observations of the CaII K-line in which the nights of 03-01-2013, 03-04-2013 (not shown), and 03-06-2013 show no measurable absorption features. However, on the nights of 03-20-2013 and 03-07-2013 an extended absorption feature centered close to the radial velocity of the $\operatorname{star}(V=$ $-7.4 \mathrm{~km} \mathrm{~s}^{-1}$ ) was present. We note that during one night's observation of this star by Holweger et al. [52] they failed to detect any circumstellar CaII K-line absorption.

3.1.3. HD 58647. HD 58647 is a very young Herbig Ae/Be star of age $<1$ Myr that has an associated dusty debris disk identified by a significant mid-IR excess [7]. However, we also note that Manoj et al. [53] have argued that it is a classical Be star. Changes in the broad photospheric NaI and OI absorption line profiles have been attributed to a timevariable circumstellar contribution [54], and Grady et al. [55] have observed similar circumstellar absorption variability (due to gas accretion) in the UV lines of MgII. Spitzer Space Telescope IR spectra recorded towards HD 58647 have revealed molecular emission from polycyclic aromatic hydrocarbons (PAHs) that are known to often populate the planet-forming disks of gas and dust around young stars [56], and $\mathrm{CO}(V=1)$ emission has been detected by Brittain et al. [57] at a velocity of $+16 \mathrm{~km} \mathrm{~s}^{-1}$.

Our CaII K-line observations shown in Figure 3 reveal a strong absorption line centered at $V \sim+20 \mathrm{~km} \mathrm{~s}^{-1}$. At a distance of 320 pc to HD 58647 this absorption component must certainly be of interstellar origin, since it has a similar absorption strength and velocity to that measured towards the nearby stars of HD 58343 and HD 57139 which are both at a comparable distance based on the $\mathrm{NaI}$ observations of Welsh et al. [58]. However, on the night of 03-01-2013 an absorption event of $\mathrm{EW}=3.5 \mathrm{~m} \AA$ at $V \sim+65 \mathrm{~km} \mathrm{~s}^{-1}$ was observed which was not seen on subsequent nights. We attribute this temporal absorption event to that of an FEB origin due to the evaporation of exocomet gas.

3.1.4. HD 108767. This nearby AOIV star located at a distance of 27 pc has only a marginal mid-IR excess $[20,22]$ and has an estimated age of $260 \mathrm{Myr}$. Photometric H- and K-band imaging observations have revealed an extended emission profile consistent with the presence of a disk of exozodiacal dust around the star [59]. Previous (single night) CaII Kline observations of this star have revealed a weak absorption centered at $V \sim 0 \mathrm{~km} \mathrm{~s}^{-1}$ with an EW $=3.1 \mathrm{~mA}$ [60], whose origin has been associated with the local Gemini interstellar cloud by Redfield and Linsky [13]. Our CaII Kline observations also reveal this interstellar absorption with a very similar EW centered at $V \sim 0 \mathrm{~km} \mathrm{~s}^{-1}$ (see Figure 4, night 03-02-2013 data). However, all four night's observations also show broad absorption events in the velocity range $V=+70$ to $+130 \mathrm{~km} \mathrm{~s}^{-1}$ which we associated with FEB events. Over the course of our observations the velocity of some of this circumstellar gas appears to move towards $V \sim 0 \mathrm{~km} \mathrm{~s}^{-1}$ and reveals itself as a time-variable absorption that affects the red wing of the interstellar absorption profile (whose total EW increases to $7.4 \mathrm{~m} \AA$ ) by the night of 03-07-2013. Such variable absorption behavior is very similar to that observed towards stars like $\beta$ Pictoris and 49 Ceti, both of which have been shown to harbor exocomets in their debris disks [15].

3.1.5. HD 9672. The circumstellar CaII K-line absorption activity associated with HD 9672 (49 Ceti) has been well described by Montgomery and Welsh [15], in which this nearby 40 Myr old AlV star has exhibited significant nightly FEB activity presumably due to the evaporation of gas from infalling exocomets. It has a well-observed dust disk [61] and is one of the few debris disks that have associated sub-mm CO emission [62]. Due to its many physical similarities to 

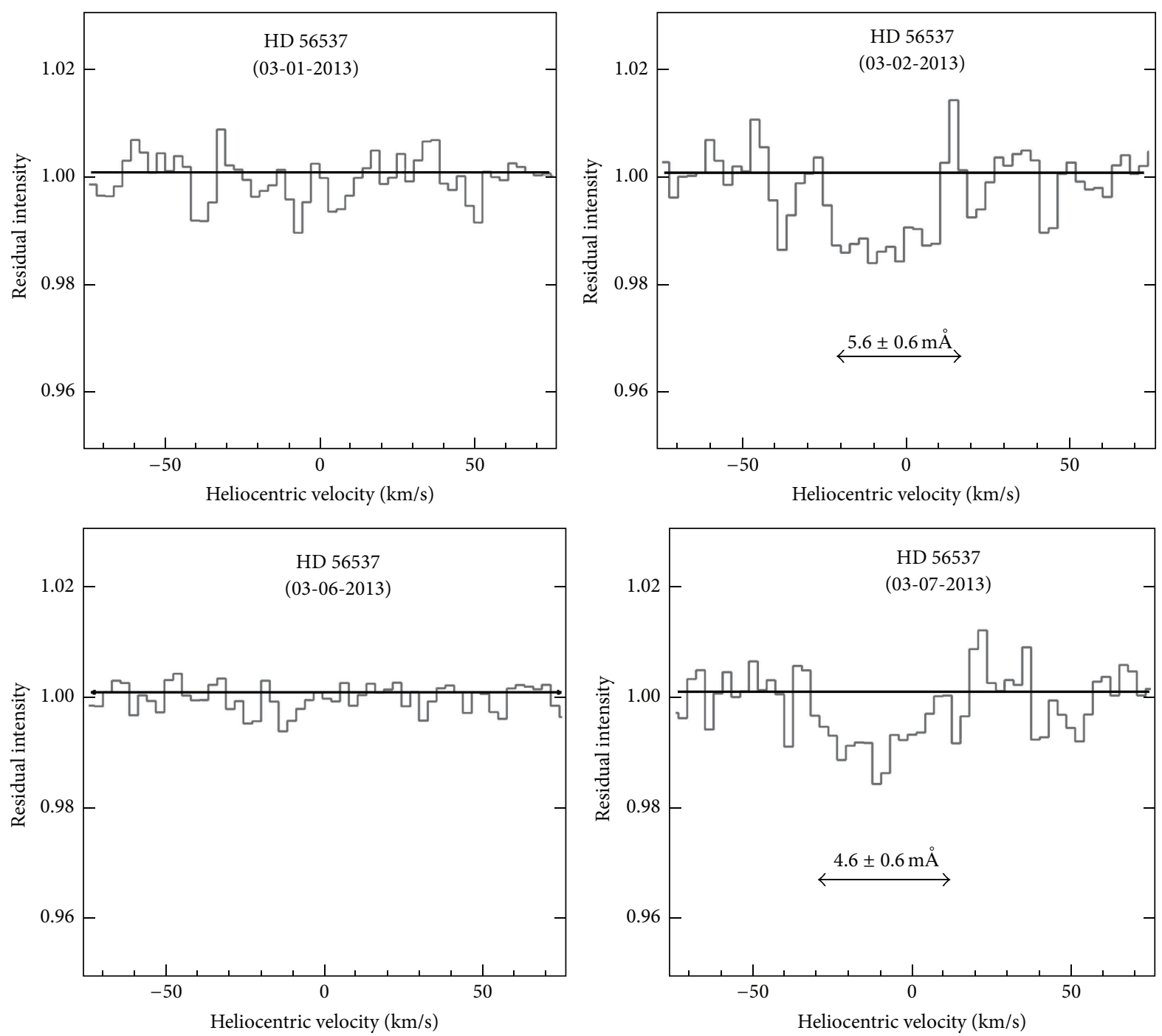

Figure 2: The circumstellar CaII K-line absorption spectra of HD 56537 recorded on 4 nights in March 2013. Note the appearance, disappearance, and reappearance of an absorption feature centered at $V \sim-10 \mathrm{~km} \mathrm{~s}^{-1}$ over a 6-day time-span.

the canonical $\beta$ Pictoris debris disk system, HD 9672 was chosen for subsequent ultraviolet absorption observations using the HST-STIS instrument over the period 08-11-162013. Preliminary results from the ultraviolet observations of the neutral absorption lines of CI and OI have been presented by Roberge et al. [46], in which (based on the derived C/Fe ratio) it appears that 49 Ceti has a volatile-rich gas disk similar in some respects to that of $\beta$ Pictoris.

In Figure 5 we show our observations of the circumstellar CaII K-line recorded three times during the night of 8-112013 and one observation on the following night of 8-12-2013. During the 2.5 hours of observations on the first night we detected an FEB event at $V \sim-35 \mathrm{~km} \mathrm{~s}^{-1}$, which disappeared by the following night only to be replaced by a new FEB absorption event at $V \sim+50 \mathrm{~km} \mathrm{~s}^{-1}$. The EW of the main CaII $\mathrm{K}$-line at $V \sim+12 \mathrm{~km} \mathrm{~s}^{-1}$ remained unchanged (within the measurement errors) throughout all of these observations. We note that the projected velocity of the local interstellar cloud of $V=11.0 \mathrm{~km} \mathrm{~s}^{-1}$ derived by Redfield and Linsky [13] is very similar to that of the radial velocity of the star, and thus the main absorption profile consists of both circumstellar and interstellar contributions. The evolution of the FEB event over the 8-11-2013 to 8-12-2013 time period in relation to the circumstellar UV absorption lines with a similar ionization potential to that of CaII will be discussed in a forthcoming paper by Roberge et al. [63].

3.1.6. HD 80007. This A1 III star, located at a distance of $35 \mathrm{pc}$, is often referred to as being "Vega-like," but Redfield et al. [64] have failed to detect a significant mid-IR excess that is normally associated with emission from a surrounding dusty debris disk. Previous observations of this star by Hempel and Schmitt [65] have shown a variation in both the EW of the CaII K-line and its absorption velocity recorded over a sixyear time period. In addition, very high spectral resolution observations of the CaII K-line by Redfield et al. [64] revealed 

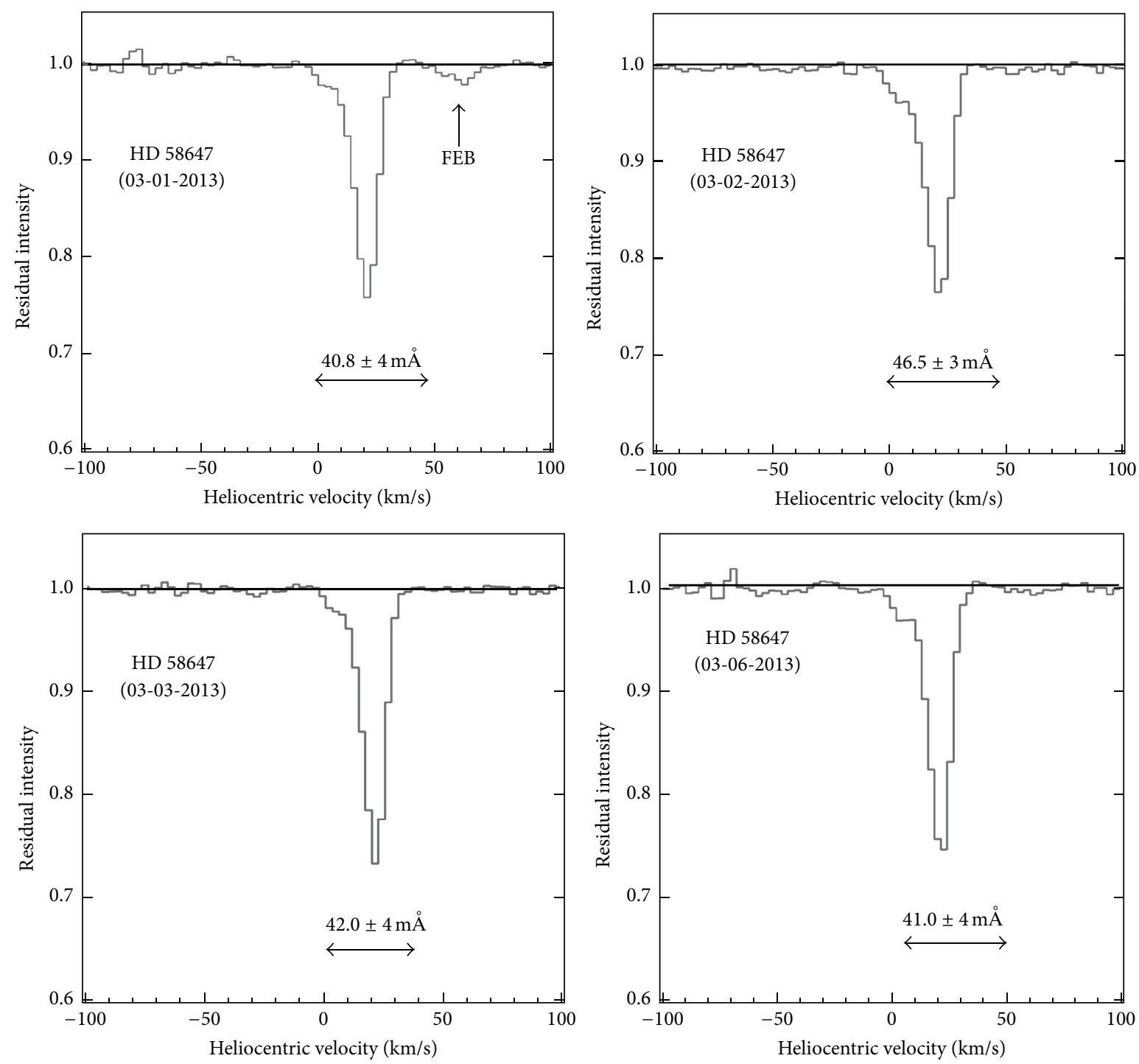

FIGURE 3: The circumstellar CaII K-line absorption spectra of HD 58647 recorded on 4 nights in March 2013. An FEB absorption event at $V \sim+65 \mathrm{~km} \mathrm{~s}^{-1}$ that is present on 03-01-2013 moves to blue wavelengths by the following night which accounts for the increase in the equivalent width of the central absorption on 03-02-2013. This "extra" absorption disappears in the spectra recorded on the following nights.

EW values varying from 0.5 to $3.1 \mathrm{~m} \AA$ recorded over a twomonth period. Both of these sets of data are in agreement with our present observations of HD 80007 shown in Figure 6. Spectra recorded on the first two nights show a broad absorption profile with a quasi two-component structure, with one component centered near the radial velocity of the star at $V \sim-3 \mathrm{~km} \mathrm{~s}^{-1}$. The latter two night's observations show only a one-component absorption with overall reduced EW, centered close to the stellar radial velocity.

This type of variable absorption behavior is unlike that of the "classic" FEB variable absorption phenomenon, such as that routinely observed towards $\beta$ Pictoris and 49 Ceti. This may be related to the advanced age of $260 \mathrm{Myr}$ for this system. We note that the Balmer line observed towards HD 80007 has been shown to be variable [66], which might suggest a stellar origin for the absorption variability we have observed. One possible scenario could be associated with mass loss accompanied by diffusion/accretion of matter with a deficit of metals from the surrounding gas/dust disk. Since the convective zone of an A-type star is extremely thin, this may lead to contamination by circumstellar and/or interstellar matter into the stellar photospheric layers. Clearly further observations of this bright and nearby star are required in order to establish whether the observed absorption variability is due to a stellar or exocomet evaporation phenomenon.

3.1.7. HD 109573. In Figure 7 we show archival spectra of the AO-type binary star HD 109573 (HR 4796A) recorded with the ESO FEROS spectrograph on 3 nights within a two-month period in 2007, together with one additional observation in 2010. This star has long been known to possess a significant IR excess [67] with the emission originating in a ring-like dusty circumstellar debris disk thought to be 



FIgURE 4: The circumstellar CaII K-line absorption spectra of HD 108767 recorded on 4 nights in March 2013. Note the appearance of the extra absorption to the main CaII line at $+10 \mathrm{~km} \mathrm{~s}^{-1}$ on 03-06-2013 and 03-07-2013. In addition an FEB absorption event with varying strength is observed on all 4 nights at $V \sim+100 \mathrm{~km} \mathrm{~s}^{-1}$.

a protocometary cloud [68]. The star has an estimated age of only $~ 8 \mathrm{Myr}[69]$ and Koerner et al. [70] have suggested that the observed morphology of the dust emission is consistent with its disk being in a transitional planet-forming stage, between that of massive gaseous protostellar disks and more tenuous debris disks such as the one detected around Vega. More recent high spatial resolution imaging of this system by Schneider et al. [71] has found that the spatial distribution of the disk grains is consistent with the possibility of one or more (as yet) unseen exoplanets, and the colors of the dust grains within the disk are consistent with a collisional evolved population of debris.

Far UV absorption observations of the gas associated with the $\mathrm{HD} 109573$ disk have failed to detect $\mathrm{H}_{2}$, and this nondetection implies that the debris disk is almost depleted of molecular gas [72]. Observations of the (weak) CaII Kline over a period of 24 hours by Welsh et al. [27] in 1997 showed no change in its absorption characteristics, which is consistent with higher resolution observations that revealed the central CaII absorption to consist of two absorption components at $V=-4$ and $-14 \mathrm{~km} \mathrm{~s}^{-1}$, both well displaced from the $V=+7.1 \mathrm{~km} \mathrm{~s}^{-1}$ radial velocity of the star [65]. The projected velocity of the local G-cloud derived by Redfield and Linsky [13] is $V=-14.9 \mathrm{~km} \mathrm{~s}^{-1}$ and thus the observed CaII K-line (unchanging) absorption is not likely to be of circumstellar origin but likely to be of interstellar origin. However, the two observations recorded on the night of 200705-04 clearly show a broad and shallow absorption feature at $V \sim+60 \mathrm{~km} \mathrm{~s}^{-1}$, which was not detected on the other two nights' observations. This is most probably an FEB absorption event, since HD 109573 is young, has an associated debris disk, and is thought to harbor exoplanet-like bodies.

3.1.8. $\beta$ Pictoris. HD 39060 ( $\beta$ Pictoris) is probably the most well-observed A-type star with an associated debris disk, exocomet activity, and at least one exoplanet. It is both young $(20 \mathrm{Myr})$ and nearby $(d=19 \mathrm{pc})$ and its debris disk 

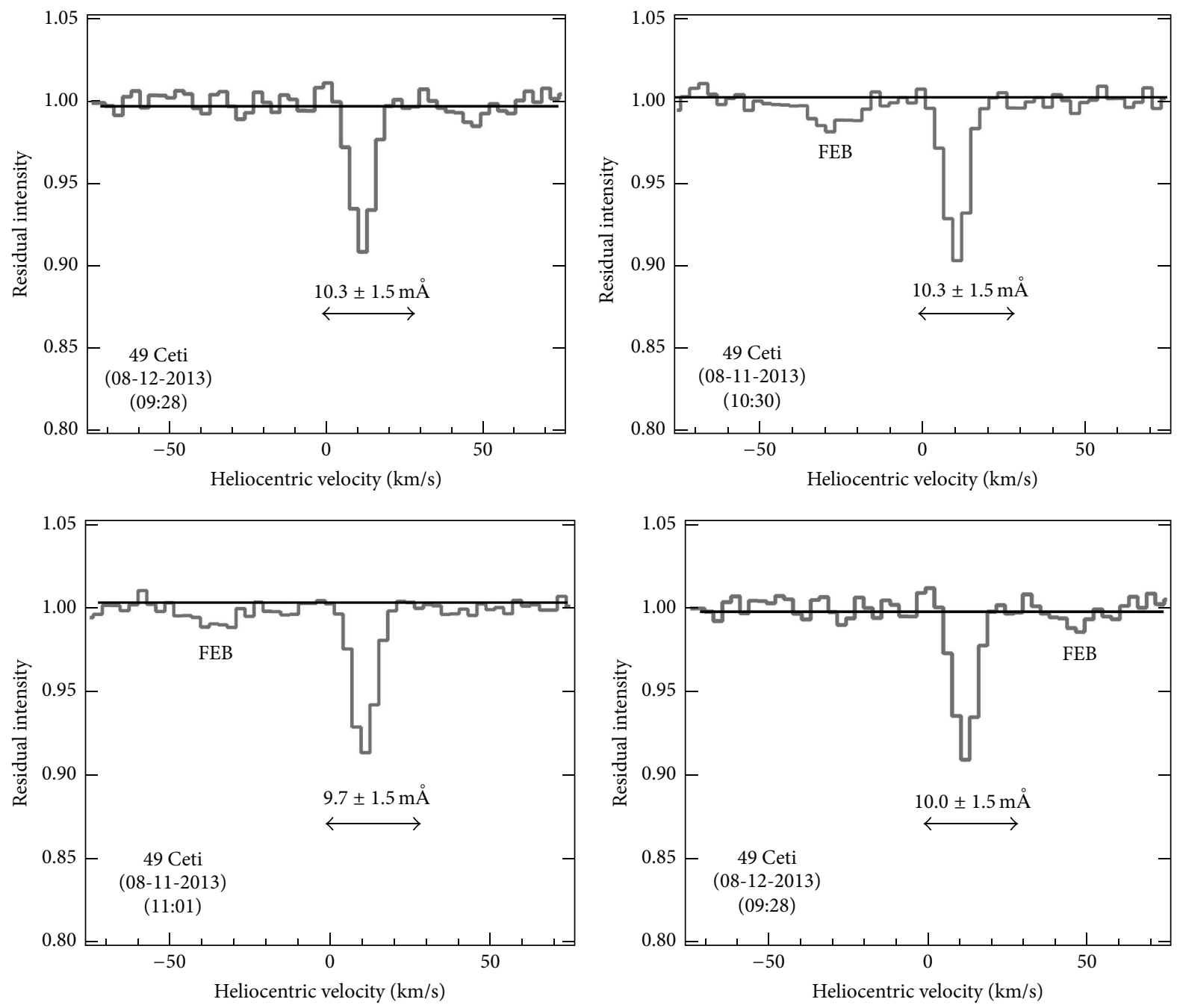

FIGURE 5: Circumstellar CaII K-line absorption recorded towards 49 Ceti (HD 9672) on the nights of 8-11-2013 and 8-12-2013. Times given in parentheses are U.T. Note the disappearance of the FEB event at $V \sim-35 \mathrm{~km} \mathrm{~s}^{-1}$ on night $8-12-2013$ and the appearance of a new FEB event at $V \sim+50 \mathrm{~km} \mathrm{~s}^{-1}$.

is viewed nearly as edge-on. A recent summary of some of the properties of the gas disk in this system can be found in present Introduction and in Kiefer et al. [73]. The vast majority of observations of the circumstellar gas in the system have focused on temporal variations of the CaII Kline $[50,74]$ in order to probe exocomet activity associated with planetesimal material in the debris disk. However, we note that studies of the disk gas surrounding $\beta$ Pictoris have also focused on the circumstellar absorption associated with $\mathrm{NaI}$ [33], CO [75], and CI [76].

Observations of the spatial structure of the optical emission from the debris disk gas by Brandeker et al. [77] and Nillson et al. [78] have revealed over 80 extended emission lines, with the brightest being FeI, NaI, Ti, and CaII. These data have been used in braking gas models by Fernández et al. [34] in order to explain the continued presence of metallic gas in the disk at fixed circumstellar velocities. Such models work best if the gas disk has an enhanced carbon FeI $\lambda 3859.91 \AA$ emission from the $\beta$ Pictoris disk that prompted us to search the ESO archive for any observations of this spectral feature in absorption. This spectral line, which arises in cold $(T<$ $500 \mathrm{~K}$ ) and neutral (ionization potential $<7.9 \mathrm{eV}$ ) gas, is very rarely seen along interstellar sightlines $[79,80]$ and thus its presence in the $\beta$ Pictoris gas disk, as first mentioned by Lagrange et al. [81], is potentially intriguing. We note that this absorption line, when present in the spectra of other A-type stars with debris disks that have been thus far observed by us, is much weaker than that found towards $\beta$ Pictoris.

In Figure 8 we show CaII K-line and FeI $\lambda 3860 \AA$ absorption spectra of $\beta$ Pictoris simultaneously recorded with the HARPS spectrograph at ESO on three nights over the 6day period 12-23-2003 to 12-29-2003. All 3 spectra show the strong circumstellar CaII K-line at $V \sim+21 \mathrm{~km} \mathrm{~s}^{-1}$, each accompanied by an FEB event of varying absorption equivalent widths with velocities in the $V=+40$ to $+60 \mathrm{~km} \mathrm{~s}^{-1}$ range. We also detect FeI absorption, centered at the circumstellar velocity of $V \sim+21 \mathrm{~km} \mathrm{~s}^{-1}$, with an (average) absorption equivalent width of $\sim 6.3 \mathrm{~m} \AA$, corresponding to a FeI column 

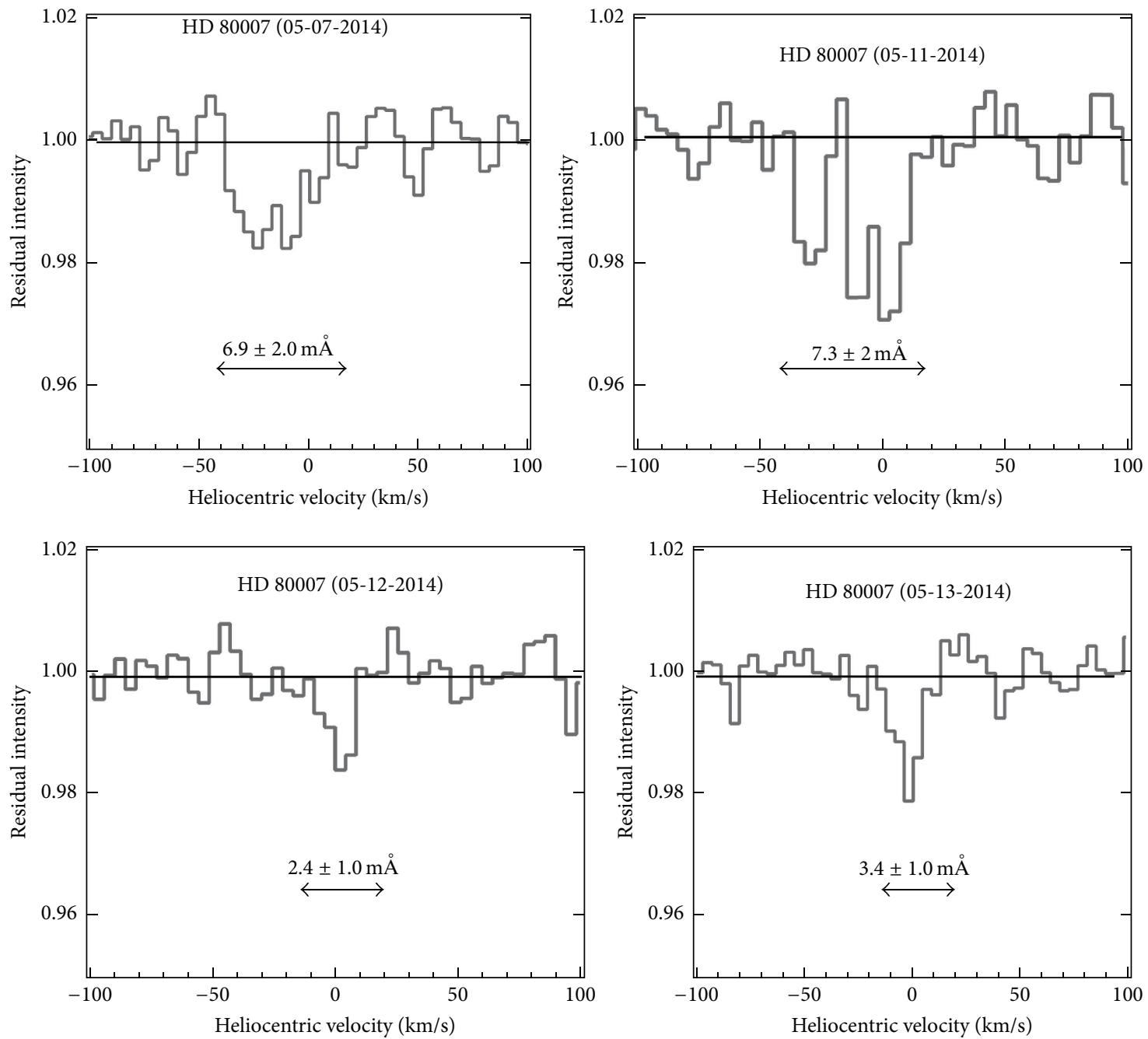

FIgURE 6: Circumstellar CaII K-line absorption spectra of the star HD 80007 recorded at SAAO over the period 05-07-2014 to 05-13-2014.

density of $\mathrm{N}(\mathrm{FeI})=2.5 \times 10^{11} \mathrm{~cm}^{-2}$. Since $\beta$ Pictoris lies well within the highly ionized region of the Local Cavity as mapped by Welsh et al. [58], this gas column density must be of circumstellar rather than of interstellar origin. We also note the appearance of what appears to be an FEB event centered at $V \sim+45 \mathrm{~km} \mathrm{~s}^{-1}$ in the FeI spectrum recorded on 12-282003. This is mirrored by an FEB event at a similar velocity simultaneously recorded in the CaII K-line spectrum of that night. To our knowledge, this is the first detection of an FEB absorption event that accompanies the FeI circumstellar line. FEB events were also recorded in the CaII K-line spectra on each of the other two nights, but these were not accompanied by similar absorption events in the corresponding FeI spectra. The FEB event in the CaII K-line spectrum of 12-28-2003 was of a far larger EW value than the other 2 nights, which may suggest that there is a CaII absorption strength threshold above which FEB events must have in order to be detected in the FeI line.

A recent analysis of over 1000 archival spectra of the circumstellar CaII $\mathrm{H}$ and $\mathrm{K}$-lines detected towards $\beta$ Pictoris by Kiefer et al. [73] has revealed the existence of two families of exocomets with different physical properties. The CaII Kline FEB absorption event recorded on 12-28-2003 and shown in Figure 8 is of type "D" (i.e., deep absorption with a narrow FWHM) as opposed to the type "S" FEB events (i.e., shallow absorption with a broad FWHM). The FeI FEB absorption event of 12-28-2003 covers a broad range of velocities (30 to $55 \mathrm{~km} \mathrm{~s}^{-1}$ ) but is of a shallow absorption depth with an $\mathrm{EW}=4.0 \mathrm{~m} \AA$ (i.e., $\mathrm{N}(\mathrm{FeI})=1.3 \times 10^{11} \mathrm{~cm}^{-2}$ ). Its broad profile suggests that it is composed of several absorption components closely spaced in velocity.

This type of absorption profile could be interpreted as originating in the evaporation of gas from several swarms of exocomets, each with similar velocities. Clearly more observations are required in order to investigate any physical relationship between FeI, CaII, and NaI circumstellar absorption. This will be accomplished in a future publication by Montgomery et al. [82] that will utilize all of the 1000+ spectra of $\beta$ Pictoris that currently reside in the ESO data archive.

3.2. Stars Showing No Variation in CaII-K Absorption. In Figure 9 we show CaII K-line absorption spectra of six stars 

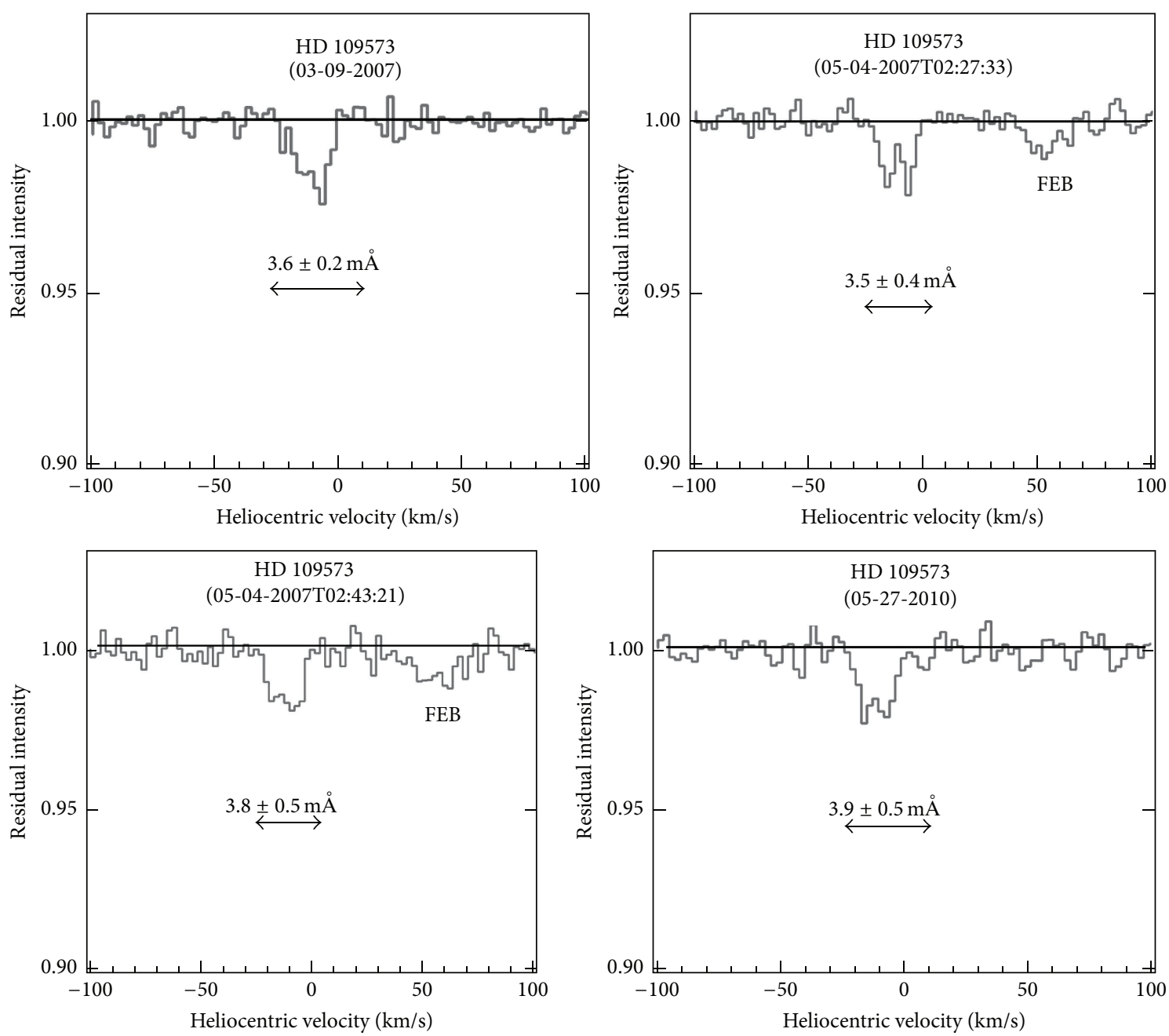

FIgURE 7: Four archival circumstellar CaII K-line absorption spectra of HD 109573 recorded with the ESO FEROS spectrograph on 3 nights within a two-month period in 2007 and one night in 2010. Note the appearance and disappearance of an FEB absorption event at $V \sim$ $+60 \mathrm{~km} \mathrm{~s}^{-1}$ that appeared in two spectra during the night of 05-04-2007.

(HD 88195, HD 106591, HD 135742, HD 158352, HD 161868, and HD 184006) that showed no significant change in the absorption strength between multiple observations typically spanning several nights. On each plot we show the measured EW value of the CaII K-line and in order to help to determine whether the observed line is of interstellar or circumstellar origin we indicate the position of the projected velocity of the most probable cloud component of local interstellar cloud complex as derived by Redfield and Linsky [13]. These data are summarized in Table 3, the last column of which indicates whether the measured absorption is most probably of interstellar (ISM) or circumstellar (C/S) origin.

For the case of HD 88195, with a distance of $183 \mathrm{pc}$, both of the components in the observed CaII K-line profile are probably dominated by absorption arising along the line-of-sight interstellar medium. However, we note that the (interstellar) absorption component at $V=+21.8 \mathrm{~km} \mathrm{~s}^{-1}$ has a very similar velocity to that of the radial velocity of the star and hence a small part of this absorption could be of circumstellar origin. The stars HD 106591, HD 135742, and HD 161868 all have distances $<60 \mathrm{pc}$ such that their sightline absorption may well be dominated by the local interstellar cloud complex. A comparison of the stellar radial velocities of these three stars, as listed in Table 3, indicates that the measured absorption can indeed be associated with that of the local interstellar clouds. Our observations of HD 106591 are consistent with those of Frisch et al. [83] who detected an (interstellar) absorption component of similar strength at $V \sim+3.8 \mathrm{~km} \mathrm{~s}^{-1}$. The present CaII K-line profile of HD 135742 is centered at a very similar velocity to the observations of Redfield and Linsky [13] who detected UV lines (of interstellar origin) at $V \sim-26.9 \mathrm{~km} \mathrm{~s}^{-1}$. We note that our observations of HD 161868 are consistent with previous (far higher resolution) observations by Crawford et al. [84] in which the CaII K-line profile was composed of two components (at $V=-30$ and $-33 \mathrm{~km} \mathrm{~s}^{-1}$ ) with a total $\mathrm{EW}=$ $6.1 \pm 0.4 \mathrm{~m} \AA$.

The CaII K-line profile of HD 158352 shown in Figure 9 shows a two-component absorption structure with the main component centered at $V \sim-28 \mathrm{~km} \mathrm{~s}^{-1}$, which is identical to 

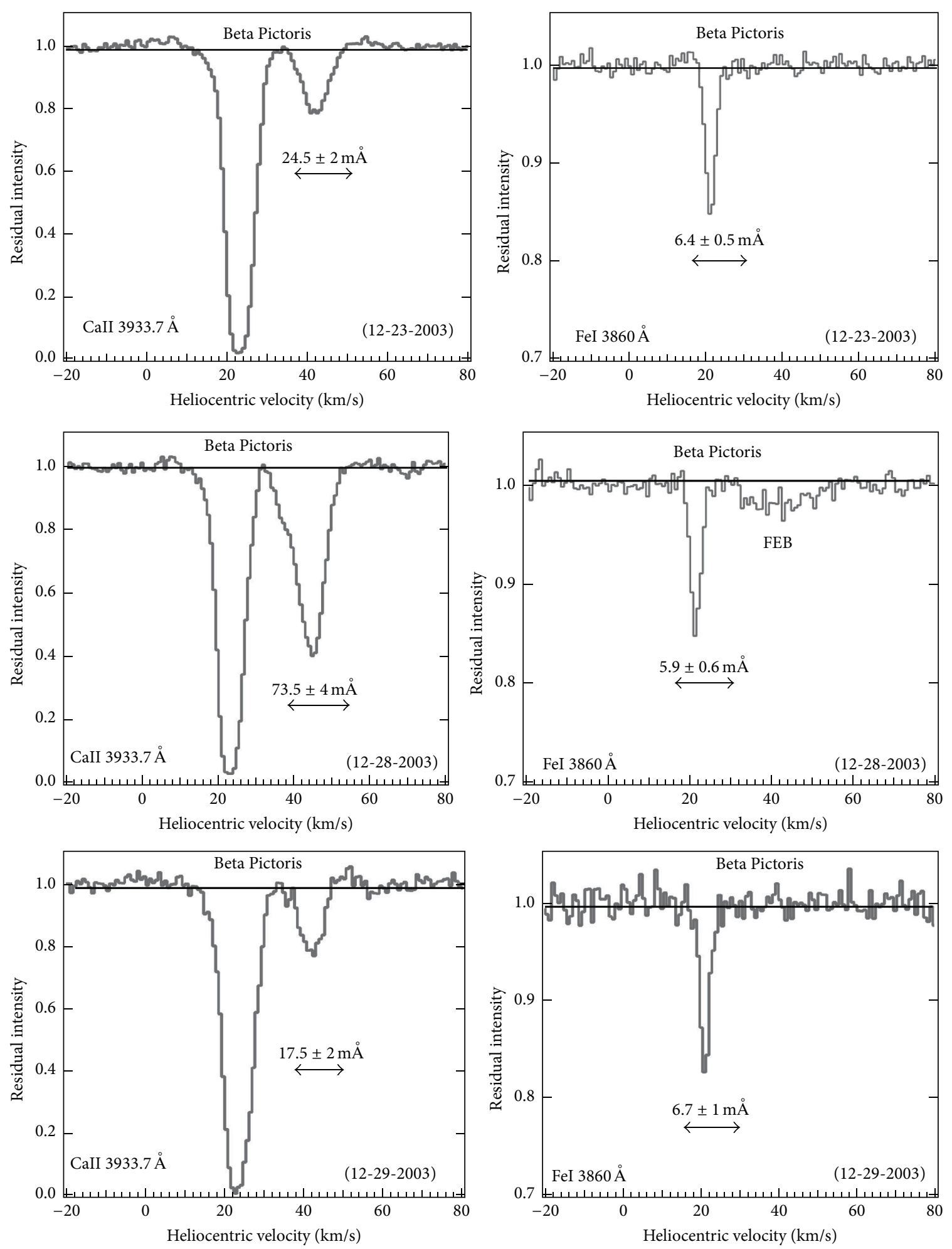

FIgUre 8: Circumstellar CaII K-line and FeI $3860 \AA$ absorption lines recorded towards $\beta$ Pictoris over a 6-day timeframe in 2003. Note the appearance of an FEB event at $V \sim+45 \mathrm{~km} \mathrm{~s}^{-1}$ in the FeI spectrum of 12-28-2003. 

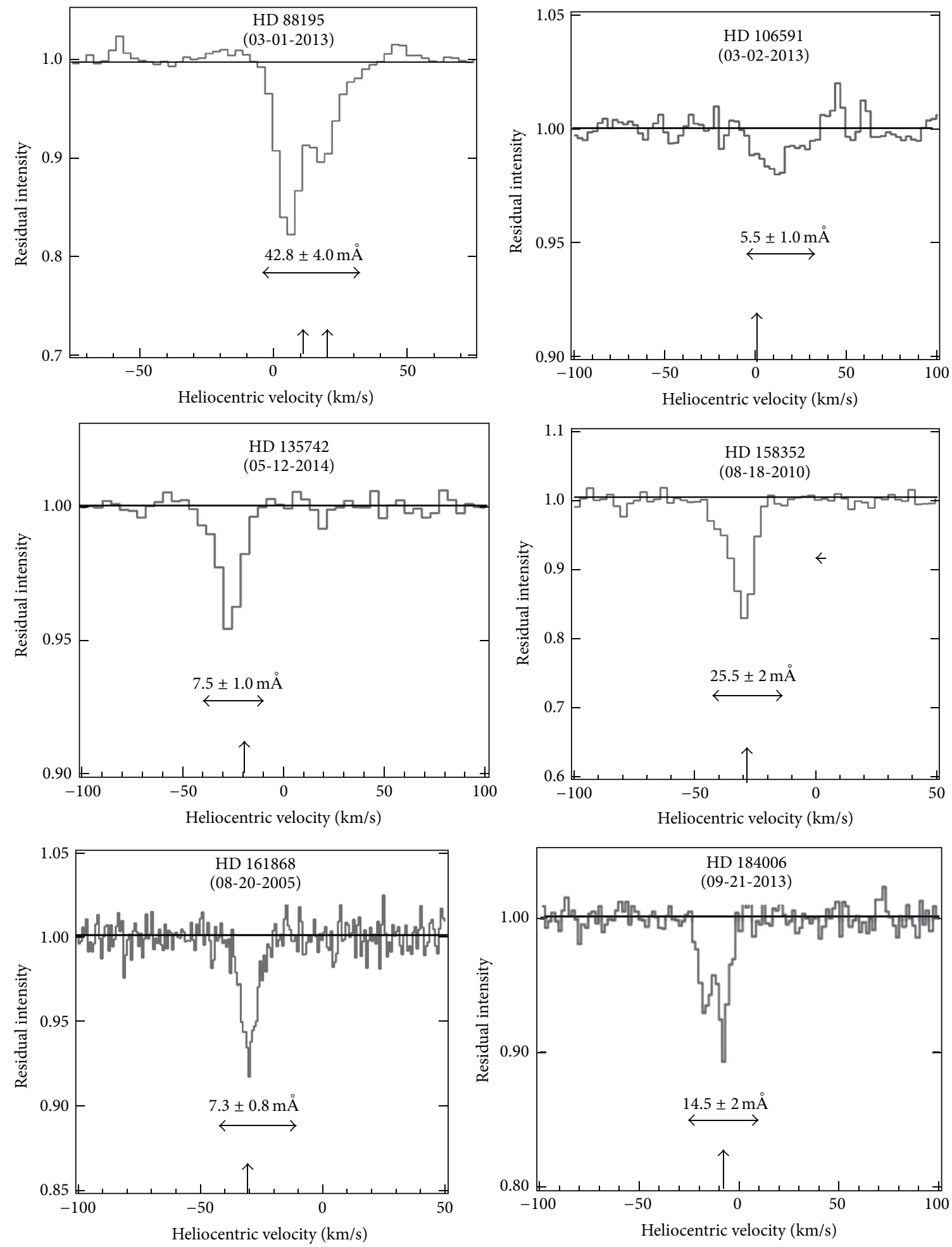

FIGURE 9: Stars showing no measurable change in their CaII K-line absorption between several nights' observations. A typical spectrum from one night is shown for each of the 6 stars, together with their absorption EW value. The vertical arrow(s) shows the position of the projected velocity of the local interstellar cloud complex derived by Redfield and Linsky [13]. 
the projected velocity of the local interstellar cloud complex listed in Table 3. However, the blue wing of the absorption profile has a component at $V \sim-37 \mathrm{~km} \mathrm{~s}^{-1}$ which is very close to the stellar radial velocity value of $-36.1 \mathrm{~km} \mathrm{~s}^{-1}$. Therefore, we identify this component as being of circumstellar origin. However, we observed no change in the strength of this circumstellar component over six nights of observation, and also no FEB absorption activity was detected.

The star HD 184006 ( $\iota$ Cyg) was observed four times over a two-week period in 2013, with all four spectra showing a two-component absorption structure (see Figure 9). The stronger of the two components has a velocity of $V \sim$ $-8.3 \mathrm{~km} \mathrm{~s}^{-1}$, which is close to the projected velocity of the local interstellar cloud complex as listed in Table 3. However, the second (and weaker) absorption component has a velocity of $V \sim-17.4 \mathrm{~km} \mathrm{~s}^{-1}$, which is close to the radial velocity, $V=-19.5 \mathrm{~km} \mathrm{~s}^{-1}$, of the star. Thus, we deem it highly likely that this gas component is circumstellar in nature. Neither of the two components changed their respective EW values over the present period of observations. However previous observations of this star with a similar spectral resolving power by us in 2010 [15] suggested that the $V=-17.4 \mathrm{~km} \mathrm{~s}^{-1}$ circumstellar component possessed a higher EW value than that recorded in 2013. In fact, the 2010 data showed that the circumstellar component was stronger than the interstellar component over the period 8-19-2010 to 8-23-2010. Those observations now fully support our contention that HD 184006 does indeed possess a circumstellar gas disk that varies in density, albeit slowly, over time.

Finally we note that although we have found no temporal variation in the absorption towards these stars (which would mostly appear to be of interstellar origin) because of the limited number and time coverage of these data we cannot totally rule out the presence of any circumstellar gas and associated FEB activity towards each of these stars.

3.3. Stars with No Measurable Circumstellar CaII K-Line Absorption. Six of the targets listed in Tables 1 and 2 (HD 31295, HD 38678, HD 71155, HD 95418, HD 112429, and HD 125161) had no measurable circumstellar CaII K-line absorption, all typically to a level of $<1.2 \mathrm{~mA}$. All six stars have distances $<38 \mathrm{pc}$, thus placing them all within the rarefied Local Cavity, which is known to be deficient in neutral gas [58]. We assume that these nondetections are due to either a low level of circumstellar gas surrounding these stars or the fact that the line-of-sight inclination angle of the debris disk is large.

\section{Discussion}

Our present research has revealed one new late B-type (HD 58647) and four new A-type stellar systems (HD 64145, HD 56537, HD 108767, and HD 109573) that exhibit short-term (night to-night) variation in their circumstellar CaII K-line absorption profiles (i.e., FEB activity). We associate such variability with the evaporation of gas from comet-like bodies on their grazing approach to a parent star. This evaporation of gas, at least for the case of $\beta$ Pictoris, takes place within
$20 R_{*}$ of the star. Although the CaII K-line profiles for HD 80007 presented in Figure 6 show circumstellar absorption variability over a six-night time frame, we are unable at present to confidently associate this absorption change with FEB activity. Additionally we have detected circumstellar CaII K-line absorption towards five other stars (HD 88195, HD 106591, HD 135742, HD 158352, and HD 161868) that showed no significant change in absorption strength between several observations. As mentioned previously, although the present (short-term) observations of HD 184006 showed no circumstellar absorption variability, a comparison with previous observations made in 2010 does indicate variability over the long term. All of our stellar targets were selected for observation on the basis of their relative youth, rapid stellar rotation, and mid-IR excess such that they possessed a known (or suspected) associated debris disk. With such similar physical properties it is therefore instructive to determine why some A-type systems with dust disks exhibit variable circumstellar gas absorption and others do not. Our previous work on this subject indicated that FEB activity tends to occur in significantly younger stellar systems that also exhibit chemical peculiarities [3]. We now update these findings using the additional data in the present paper.

In the upper half of Table 4 we list 13 A-type stars (with relevant references) that have observed circumstellar CaII Kline absorption but do not exhibit any significant short-term absorption variability when measured over several nights' observations. Since circumstellar CaII K-line absorption can often be confused with (local) interstellar absorption with a similar velocity, we deem an absorption component to be of circumstellar origin if it lies within $\pm 6 \mathrm{~km} \mathrm{~s}^{-1}$ of the radial velocity of the central star (i.e., one spectral resolution element) and its velocity is inconsistent with that of the projected velocity of the local interstellar cloud complex as derived by Redfield and Linsky [13]. In the lower part of Table 4 we list (16) stars that do possess short-term (night-tonight) circumstellar absorption variability of the CaII K-line profile that has been associated with FEB activity. Note that our list of stars does not include the A0-type star HD 32297 which has displayed variable circumstellar $\mathrm{NaI}$ absorption but no CaII K-line variability [85] nor does it include the AOV-type star HD 158643 (51 Oph) in which Hempel and Schmitt [65] suspect circumstellar CaII K-line variability but has yet to show FEB activity.

In addition to the spectral type, age, and mid-IR excess data in Table 4, column 6 lists whether the star was identified as chemically peculiar (CP) in the work of Zorec and Royer [86]. In column 7 we identify stars labeled as $\lambda$ Bootis $[87,88]$, and in column 8 we list the equivalent width value of the $\lambda 4481 \AA \mathrm{MgII}$ absorption line as measured by Abt and Morrell [89]. These stars are characterized by an underabundance of elements such as $\mathrm{Mg}, \mathrm{Ca}$, and $\mathrm{Fe}$, such that a weakness of the MgII line is generally adopted as a primary criterion for the classification of $\lambda$ Bootis stars.

Firstly all of the 29 targets listed in Table 4 possess a circumstellar gas disk, as indicated by the presence of the CaII K-line near the radial velocity of the central star. We note that 12 of the 29 stars in Table 4 have been classified as 
shell-type [90], which by definition implies the presence of circumstellar gas surrounding the central star. The majority of those stars with known ages of $<100 \mathrm{Myr}$ also possess debris (or transitional) disks, as determined either by their observed mid-IR excess or by direct high-resolution imaging studies in the IR. Hence, just based on these two factors alone we might expect that such systems would in addition to a gas disk that is presumably located close to the central star also possess primordial objects such as planetesimals, comets, and asteroids that are associated with the more distant debris disk.

A statistical analysis of the (unfortunately incomplete) data listed in Table 4 reveals the following results:

(1) Although the FEB-hosting stars are on average $70 \mathrm{Myr}$ younger than the non-FEB-hosting stars, there are only $78 \%$ chances that this difference is actually real. Also deriving accurate ages for A-type stars is problematic, with several of the ages listed in Table 4 being obtained from cluster membership rather than by individual stellar observations.

(2) There is no statistical difference between the two sets of stars based on either their mid-IR excess or their rotational velocities.

(3) As opposed to our previous finding there is no highly statistical difference between the two sets of stars based on either their metallicity or their chemical peculiarity (even if we include $\lambda$ Bootis stars).

(4) One interesting difference between the two sets of stars seems to be that FEB-hosting stars are of an earlier type (on average A1.8 as compared with A3.1). A $t$-test reveals that there is an $88 \%$ chance that the FEB-hosting stars are of an earlier type. This could be due to stellar activity levels (such as chromospheric activity or nonradial pulsations) being higher within the atmospheres of hotter A-type stars.

Finally we note that the detection of FEB events is critically dependent on two observational factors that are independent of any of the stellar parameters listed in Table 4. Firstly the viewing angle of the gas disk must be close to being edge-on for an observer to detect a strong absorption signal from the circumstellar gas and also any changes in the CaII $\mathrm{K}$-line absorption profile due to FEB activity. Secondly, our observations generally span timeframes of only one week, and thus A-type debris disk systems that have lower levels of FEB activity would be incorrectly categorized in our Table 4 .

\section{Conclusion}

We have presented medium resolution $(R \sim 60,000)$ spectral observations of the CaII K-line $(3933 \AA)$ recorded in absorption towards 15 A-type stars (plus two late B-type stars) with known (or suspected) surrounding debris disk. In addition, we have presented similar spectral absorption data for three A-type stars that were retrieved from the European Southern Observatory on-line data archive. Nightly changes in the CaII K-line absorption profile were observed for five stars (HD56537, HD 58647, HD 64145, HD 108767, and HD
109573). This type of absorption variability that is observed in A-type stars with associated debris disks is thought to originate in a circumstellar gas disk surrounding such young stellar systems. The weak absorption features that sporadically appear within $\pm 100 \mathrm{~km} \mathrm{~s}^{-1}$ of the central circumstellar CaII K-line absorption profile are most probably associated with the presence of "falling evaporating bodies" (FEBs or exocomets) that liberate evaporative gas on their grazing trajectory towards and around the central star. It is widely believed that planets form through a long period of impacts, mergers, and the accretion of kilometer-sized planetesimals, which themselves have been formed from the accretion of dust grains formed from heavy elements condensing out gaseous circumstellar disks $[9,91]$. Our present detection of gas liberated from such bodies that are thought to be the building blocks of planetary systems fully supports current theoretical models of (exo)planetary formation. We note that the detection of such short-term exocomet activity around these young $(<100 \mathrm{MYr})$ stars may currently be the best indication of the presence of associated (but as yet unobserved) exoplanets orbiting these young stellar systems. Hence, we urge further observations of the 16 stars exhibiting FEB activity listed in Table 16 using high precision imaging techniques such as that used by Lagrange et al. [17] in the successful detection of the $\beta$ Pictoris $b$ exoplanet.

We have also presented archival spectra of the circumstellar FeI $\lambda 3860 \AA$ and CaII K-lines observed towards $\beta$ Pictoris (HD 39060) that, for the first time, reveal the absorption signatures of FEB activity at similar velocities in both of the line profiles. Although we have only made a cursory inspection of the 1000+ spectra residing in the European Southern Observatory (ESO) data archive, our preliminary finding is that an FEB absorption event is only detected in the FeI line whenever a similar FEB absorption event is observed with an equivalent width value of $>50 \mathrm{~m} \AA$ in the CaII Kline profile. Recent findings, based on observations of the circumstellar CaII K-line, suggest that there are at least two families of exocomets with different physical properties in orbit around $\beta$ Pictoris [73]. Thus, it would be interesting to investigate any physical relationship between FeI, CaII, and $\mathrm{NaI}$ circumstellar absorption in this young disk system. This will be accomplished in a future publication by Montgomery et al. [82] that will use all of the $1000+$ spectra of $\beta$ Pictoris that currently reside in the ESO data archive.

Finally we attempted to determine which, if any, of the stellar physical parameters of young debris disk systems determine whether FEB activity (as defined by the sporadic presence of weak absorption features in the circumstellar CaII $\mathrm{K}$-line profile) is present. None of the parameters, such as rotational velocity, mid-IR excess, or chemical peculiarity, seem to differentiate between stars in which FEB activity is present and those in which it is not observed. Our data suggests that FEB-hosting stars are of an earlier type (on average spectral type Al.8 as compared with A3.1) than those in which no FEB activity has been observed. This could be due to stellar activity levels (such as chromospheric activity or nonradial pulsations) being of a higher level within the atmospheres of these hotter A-type stars. Finally it would 
appear that stellar age does not seem to be a significant factor in determining the presence of exocomet activity around A-type stars. This is in conflict with most predictions of planetary system formation and may well be due to the inherent uncertainty in the derivation of the ages we have listed for the stars in Table 4.

\section{Conflict of Interests}

The authors declare that there is no conflict of interests regarding the publication of this paper.

\section{Acknowledgments}

The authors particularly acknowledge the assistance of the dedicated staff at the McDonald Observatory, the Pie du Midi Observatory, and the South African Astronomical Observatory. Both Ben Bukoski and Sarah Strausbaugh helped with the data extraction and the authors appreciate the work carried out by Rosine Lallement in accessing the NARVAL data for them. Finally they acknowledge the unerring support of the amazing $\mathrm{C}^{2}$.

\section{References}

[1] A. Fortier, Y. Alibert, F. Carron, W. Benz, and K.-M. Dittkrist, "Planet formation models: the interplay with the planetesimal disc," Astronomy \& Astrophysics, vol. 549, article A44, 19 pages, 2013.

[2] P. Garaud, F. Meru, M. Galvagni, and C. Olczak, "From dust to planetesimals: an improved model for collisional growth in protoplanetary disks," The Astrophysical Journal, vol. 764, no. 2, article 146, 2013.

[3] B. Y. Welsh and S. Montgomery, "Circumstellar Gas-Disk variability around A-type stars: the detection of exocomets?" Publications of the Astronomical Society of the Pacific, vol. 125, no. 929, pp. 759-774, 2013.

[4] J. H. Debes, M. Kilic, F. Faedi et al., "Detection of weak circumstellar gas around the DAZ white dwarf WD 1124293: evidence for the accretion of multiple asteroids," The Astrophysical Journal, vol. 754, no. 1, p. 59, 2012.

[5] B. Zuckerman and I. Song, "A 40 Myr old gaseous circumstellar disk at 49 Ceti: massive co-rich comet clouds at young A-type stars," The Astrophysical Journal, vol. 758, no. 2, p. 77, 2012.

[6] M. C. Wyatt, "Evolution of debris disks," Annual Review of Astronomy and Astrophysics, vol. 46, pp. 339-383, 2008.

[7] K. Y. L. Su, G. H. Rieke, J. A. Stansberry et al., "Debris disk evolution around a stars," Astrophysical Journal, vol. 653, no. 1, pp. 675-689, 2006.

[8] F. Morlaes, G. Rieke, M. Werner, G. Bryden, K. R. Stapelfeldt, and K. Y. L. Su, "Common warm dust temperatures around main-sequence stars," The Astrophysical Journal Letters, vol. 730, no. 2, p. L29, 2011.

[9] S. J. Kenyon and B. C. Bromley, "Detecting the dusty debris of terrestrial planet formation," Astrophysical Journal, vol. 602, no. 2, pp. L133-L136, 2004.

[10] L. M. Hobbs, A. Vidal-Madjar, R. Ferlet, C. E. Albert, and C. Gry, "The gaseous component of the disk around Beta Pictoris," The Astrophysical Journal, vol. 293, pp. L29-L33, 1985.
[11] R. Ferlet, A. Vidal-Madjar, and L. Hobbs, "The beta pictoris circumstellar disk. V-time variations of the CA II-K line," Astronomy \& Astrophysics, vol. 185, no. 1-2, pp. 267-270, 1987.

[12] A. Carmona, "Observational diagnostics of gas in protoplanetary disks," Earth, Moon and Planets, vol. 106, no. 2, pp. 71-95, 2010.

[13] S. Redfield and J. L. Linsky, "The structure of the local interstellar medium. IV. Dynamics, morphology, physical properties, and implications of cloud-cloud interactions," The Astrophysical Journal, vol. 673, no. 1, pp. 283-314, 2008.

[14] F. Kiefer, A. Lecavelier des Etangs, J.-C. Augereau, A. VidalMadjar, A.-M. Lagrange, and H. Beust, "Exocomets in the circumstellar gas disk of HD 172555," Astronomy and Astrophysics, vol. 561, article L10, 2014.

[15] S. Montgomery and B. Y. Welsh, "Detection of variable gaseous absorption features in the debris disks around young A-type stars," Publications of the Astronomical Society of the Pacific, vol. 124, pp. 1042-1056, 2012.

[16] H. Beust, A. Vidal-Madjar, R. Ferlet, and A. M. LagrangeHenri, "The Beta Pictoris circumstellar disk. X-numerical simulations of infalling evaporating bodies," Astronomy \& Astrophysics, vol. 236, no. 1, pp. 202-216, 1990.

[17] A.-M. Lagrange, M. Desort, F. Galland, S. Udry, and M. Mayor, "Extrasolar planets and brown dwarfs around A-F type stars: VI. High precision RV survey of early type dwarfs with HARPS," Astronomy \& Astrophysics, vol. 495, no. 1, pp. 335-352, 2009.

[18] E. Nielson, M. Liu, Z. Wahhaj et al., "The Gemini NICI PlanetFinding Campaign: the frequency of giant planets around young B and A stars," The Astrophysical Journal, vol. 776, no. 1, p. 4, 2013.

[19] C. H. Chen, B. M. Patten, M. W. Werner et al., "A spitzer study of dusty disks around nearby, young stars," Astrophysical Journal Letters, vol. 634, no. 2, pp. 1372-1384, 2005.

[20] G. H. Rieke, K. Y. L. Su, J. A. Stansberry et al., "Decay of planetary debris disks," The Astrophysical Journal, vol. 620, no. 2, pp. 1010-1026, 2005.

[21] J. H. Rhee, I. Song, B. Zuckerman, and M. McElwain, "Characterization of dusty debris disks: the IRAS and Hipparcos catalogs," Astrophysical Journal, vol. 660, no. 2, pp. 1556-1571, 2007.

[22] B. Montesinos, C. Eiroa, A. Mora, and B. Merín, "Parameters of Herbig Ae/Be and Vega-type stars," Astronomy \& Astrophysics, vol. 495, no. 3, pp. 901-917, 2009.

[23] D. R. Rodriguez and B. Zuckerman, "Binaries among debris disk stars," The Astrophysical Journal, vol. 745, no. 2, article 147, 2012.

[24] L. Vican, "Age determination for 346 nearby stars in the herschel DEBRIS survey," The Astronomical Journal, vol. 143, no. 6, article 135, 2012.

[25] G. A. Gontcharov, "Pulkovo compilation of radial velocities for 35495 hipparcos stars in a common system," Astronomy Letters, vol. 32, no. 11, pp. 759-771, 2006.

[26] C. Chen, T. Mittal, M. Kuehner et al., "The Spitzer infrared spectrograph debris disk catalog. I. Continuum analysis of unresolved targets," The Astrophysical Journal Supplement Series, vol. 211, no. 2, p. 25, 2014.

[27] B. Y. Welsh, N. Craig, I. A. Crawford, and R. J. Price, " $\beta$ Piclike circumstellar disk gassurrounding HR 10 and HD 85905," Astronomy and Astrophysics, vol. 338, no. 2, pp. 674-682, 1998.

[28] A. M. Lagrange-Henri, H. Beust, R. Ferlet, A. Vidal-Madjar, and L. M. Hobbs, "HR 10-a new Beta Pictoris-like star," Astronomy \& Astrophysics, vol. 227, no. 1, pp. L13-L16, 1990. 
[29] T. Currie, C. Thalmann, S. Matsumara, N. Madhusudhan, A. Burrows, and M. Kuchner, "A $5 \mu \mathrm{m}$ image of $\beta$ pictoris b at a sub-jupiter projected separation: evidence for a misalignment between the planet and the inner, warped disk," The Astrophysical Journal, vol. 736, no. 2, article L33, 2011.

[30] A. Lagrange and G. Chauvin, "Beta pictoris, a laboratory for planetary formation studies," The Messenger, vol. 150, pp. 39-43, 2012.

[31] L. M. Hobbs, D. E. Welty, A.-M. Lagrange-Henri, R. Ferlet, and A. Vidal-Madjar, "The location of the CA II ions in the beta Pictoris disk," The Astrophysical Journal, vol. 334, no. 1, pp. L41L44, 1988.

[32] D. Mouillet and A.-M. Lagrange, "The Beta pictoris circumstellar disk. Some physical parameters of the gaseous component," Astronomy \& Astrophysics, vol. 297, p. 175, 1995.

[33] A. Vidal-Madjar, R. Ferlet, L. M. Hobbs, C. Gry, and C. E. Albert, "The circumstellar gas cloud around Beta Pictoris II," Astronomy and Astrophysics, vol. 167, pp. 325-332, 1986.

[34] R. Fernández, A. Brandeker, and Y. Wu, "Braking the gas in the $\beta$ pictoris disk," The Astrophysical Journal, vol. 643, no. 1, pp. 509-522, 2006

[35] G. Galazutdinov, J. Krełowski, Y. Beletsky, and G. Valyavin, "Variable interstellar lines in spectra of HD 73882," Publications of the Astronomical Society of the Pacific, vol. 125, no. 933, pp. 1329-1335, 2013.

[36] L. Churcher, M. Wyatt, G. Duchene et al., "Multiwavelength modelling of the $\beta$ Leo debris disc: one, two or three planetesimal populations?" Monthly Notices of the Royal Astronomical Society, vol. 417, no. 3, pp. 1715-1734, 2011.

[37] G. Schneider, B. A. Smith, E. E. Becklin et al., "NICMOS imaging of the HR 4796A circumstellar disk," Astrophysical Journal, vol. 513, no. 2, pp. L127-L130, 1999.

[38] P. Kalas, J. Larwood, B. A. Smith, and A. Schultz, "Rings in the planetesimal disk of $\beta$ Pictoris," The Astrophysical Journal, vol. 530, no. 2, pp. 1133-1137, 2000.

[39] O. Absil, D. Defrere, V. Coudé du Foresto et al., "A near-infrared interferometric survey of debris-disc stars III. First statistics based on 42 stars observed with CHARA/FLUOR," Astronomy \& Astrophysics, vol. 555, article A104, 2013.

[40] H. Holweger, I. Rentzsch-Holm, and H. Holweger, AA, vol. 303, p. 819, 1995.

[41] K.-P. Cheng and J. E. Neff, "Far-ultraviolet observations of the circumstellar gas in the 2 andromedae system," The Astronomical Journal, vol. 125, no. 2, pp. 868-874, 2003.

[42] C. Catala, J. Czarny, P. Felenbok, A. Talavera, and P. S. The, "Short-term variability of the CA II K line in the pre-main sequence Herbig AE start HD 250550," Astronomy \& Astrophysics, vol. 244, no. 1, pp. 166-180, 1991.

[43] D. de Winter, C. A. Grady, M. E. van den Ancker, M. R. Pérez, and C. Eiroa, "Episodic accretion around the Herbig Ae star BF Orionis: evidence for the presence of extra-solar comets," Astronomy \& Astrophysics, vol. 343, no. 1, pp. 137-150, 1999.

[44] N. Mariñas, C. M. Telesco, R. S. Fisher, and C. Packham, "Highresolution mid-infrared imaging of the circumstellar disks of HERBIG Ae/Be stars," The Astrophysical Journal, vol. 737, no. 2, p. 57, 2011.

[45] N. D. Thureau, J. S. Greaves, B. C. Matthews et al., "An unbiased study of debris discs around A-type stars with Herschel," Monthly Notices of the Royal Astronomical Society, vol. 445, no. 3, pp. 2558-2573, 2014.
[46] A. Roberge, B. Y. Welsh, I. Kamp et al., "Volatile-rich circumstellar gas in the unusual 49 Ceti debris disk," The Astrophysical Journal Letters, vol. 796, no. 1, article L11, 2014.

[47] K. Horne, "An optimal extraction algorithm for CCD spectroscopy," Publications of the Astronomical Society of the Pacific, vol. 98, no. 604, pp. 609-617, 1986.

[48] J. V. Vallerga, P. W. Vedder, N. Craig, and B. Y. Welsh, "Highresolution Ca II observations of the local interstellar medium," Astrophysical Journal, vol. 411, no. 2, pp. 729-749, 1993.

[49] H. Fujiwara, D. Ishihara, T. Onaka et al., "AKARI/IRC $18 \mu \mathrm{m}$ survey of warm debris disks," Astronomy \& Astrophysics, vol. 550, article A45, 15 pages, 2013.

[50] A. M. Lagrange-Henri, E. Gosset, H. Beust, R. Ferlet, and A. Vidal-Madjar, "The beta pictoris circumstellar disk. XIIIsurvey of the variable CA II lines," Astronomy \& Astrophysics, vol. 264, no. 2, pp. 637-653, 1992.

[51] R. Smith and M. C. Wyatt, "Warm dusty discs: exploring the A star $24 \mu \mathrm{m}$ debris population," Astronomy \& Astrophysics, vol. 515, article A95, 2010.

[52] H. Holweger, M. Hempel, and I. Kamp, "A search for circumstellar gas around normal A stars and Lambda Bootis stars," Astronomy \& Astrophysics, vol. 350, no. 2, pp. 603-611, 1999.

[53] P. Manoj, G. Maheswar, and H. C. Bhatt, "Non-emission-line young stars of intermediate mass," Monthly Notices of the Royal Astronomical Society, vol. 334, no. 2, pp. 419-425, 2002.

[54] I. Mendigutia, C. Eiroa, B. Montesinos et al., "Optical spectroscopic variability of Herbig Ae/Be stars," Astronomy \& Astrophysics, vol. 529, article A34, 2011.

[55] C. Grady, M. Perez, A. Talavera et al., "The $\beta$ Pictoris phenomenon among Herbig Ae/Be stars. UV and optical high dispersion spectra," Astronomy \& Astrophysics Supplement, vol. 120, pp. 157-177, 1996.

[56] B. Acke, J. Bouwman, A. Juhász et al., "Spitzer's view on aromatic and aliphatic hydrocarbon emission in Herbig Ae stars," The Astrophysical Journal, vol. 718, no. 1, pp. 558-574, 2010.

[57] S. D. Brittain, T. Simon, J. R. Najita, and T. W. Rettig, "Warm gas in the inner disks around young intermediate-mass stars," The Astrophysical Journal, vol. 659, no. 1, article 685, 2007.

[58] B. Y. Welsh, R. Lallement, J.-L. Vergely, and S. Raimond, "New 3D gas density maps of $\mathrm{NaI}$ and $\mathrm{CaII}$ interstellar absorption within 300 pc," Astronomy and Astrophysics, vol. 510, no. 1, article A54, 2010.

[59] S. Ertel, O. Absil, D. Defrere et al., "A near-infrared interferometric survey of debris-disk stars. IV. An unbiased sample of 92 southern stars observed in $H$ band with VLTI/PIONIER," Astronomy \& Astrophysics, vol. 570, article A128, 2014.

[60] D. E. Welty, D. C. Morton, and L. M. Hobbs, "A high-resolution survey of interstellar Ca II absorption," The Astrophysical Journal Supplement Series, vol. 106, p. 533, 1996.

[61] H. J. Walker and R. D. Wolstencroft, "Cool circumstellar matter around nearby main-sequence stars," Publications of the Astronomical Society of the Pacific, vol. 100, no. 634, pp. 1509$1521,1988$.

[62] W. R. F. Dent, J. S. Greaves, and I. M. Coulson, "CO emission from discs around isolated HAeBe and Vega-excess stars," Monthly Notices of the Royal Astronomical Society, vol. 359, no. 2, pp. 663-676, 2005.

[63] A. Roberge, B. Y. Welsh, I. Kamp, A. Weinberger, and C. Grady, The Astrophyscial Journal. In preparation.

[64] S. Redfield, J. E. Kessler-Silacci, and L. A. Cieza, "Spitzer limits on dust emission and optical gas absorption variability around 
nearby stars with edge-on circumstellar disk signatures," The Astrophysical Journal, vol. 661, pp. 944-971, 2007.

[65] M. Hempel and J. H. M. M. Schmitt, "High resolution spectroscopy of circumstellar material around A stars," Astronomy \& Astrophysics, vol. 408, no. 3, pp. 971-979, 2003.

[66] J. Wood and J. Hollis, "Balmer-line variability of Beta Carinae," Astronomy \& Astrophysics, vol. 12, p. 468, 1971.

[67] M. Jura, “The dust debris around HR 4796," Astrophysical Journal, vol. 383, no. 2, pp. L79-L80, 1991.

[68] M. Jura, M. Malkan, R. White, C. Telesco, R. Pina, and R. S. Fisher, "A protocometary cloud around HR 4796A?" Astrophysical Journal, vol. 505, no. 2, pp. 897-902, 1998.

[69] J. Stauffer, L. Hartmann, and D. Barrado y Navascues, "An age estimate for the beta pictoris analog HR 4796A," The Astrophysical Journal, vol. 454, p. 910, 1995.

[70] D. W. Koerner, M. E. Ressler, M. W. Werner, and D. E. Backman, "Mid-infrared imaging of a circumstellar disk around HR 4796: mapping the debris of planetary formation," The Astrophysical Journal Letters, vol. 503, no. 1, pp. L83-L87, 1998.

[71] G. Schneider, A. J. Weinberger, E. E. Becklin, J. H. Debes, and B. A. Smith, "Stis imaging of the HR 4796A circumstellar debris ring," Astronomical Journal, vol. 137, no. 1, pp. 53-61, 2009.

[72] C. Martin-Zaïdi, M. Deleuil, J. le Bourlot et al., "Molecular hydrogen in the circumstellar environments of Herbig Ae/Be stars probed by FUSE," Astronomy and Astrophysics, vol. 484, no. 1, pp. 225-239, 2008.

[73] F. Kiefer, A. Lecavelier des Etangs, J. Boisseir et al., "Two families of exocomets in the $\beta$ Pictoris system," Nature, vol. 514, pp. 462464, 2014.

[74] I. A. Crawford, H. Beust, and A. M. Lagrange, "Detection of a strong transient blueshifted absorption component in the $\beta$ Pictoris disc," Monthly Notices of the Royal Astronomical Society, vol. 294, no. 2, pp. L31-L34, 1998.

[75] W. R. F. Dent, M. C. Wyatt, A. Roberge et al., "Molecular gas clumps from the destruction of icy bodies in the $\beta$ pictoris debris disk," Science, vol. 343, no. 6178, pp. 1490-1492, 2014.

[76] A. Roberge, P. D. Feldman, A. M. Lagrange et al., "Highresolution Hubble space telescope STIS spectra of C I and CO in the $\beta$ pictoris circumstellar disk," Astrophysical Journal, vol. 538, no. 2, pp. 904-910, 2000.

[77] A. Brandeker, R. Liseau, G. Olofsson, and M. Fridlund, "The spatial structure of the $\beta$ pictoris gas disk," Astronomy \& Astrophysics, vol. 413, no. 2, pp. 681-691, 2004.

[78] R. Nillson, A. Brandeker, G. Olofsson, K. Fathi, P. Thébault, and R. Liseau, "VLT imaging of the $\beta$ pictoris gas disk," Astronomy \& Astrophysics, vol. 544, article A134, 2012.

[79] A. Bandar, M. Kozak, P. Gnacinski et al., "CaFe interstellar clouds," Monthly Notices of the Royal Astronomical Society, vol. 378, p. 89, 2007.

[80] Y. Ihara, J. Ozaki, M. Doi et al., "Searching for a companion star of Tycho's type Ia supernova with optical spectroscopic observations," Publications of the Astronomical Society of Japan, vol. 59, no. 4, pp. 811-826, 2007.

[81] A. M. Lagrange, A. Vidal-Madjar, M. Deleuil et al., "The $\beta$ Pictoris circumstellar disk. XVII. Physical and chemical parameters of the disk," Astronomy \& Astrophysics, vol. 296, pp. 499-508, 1995.

[82] S. Montgomery, B. Y. Welsh, B. Bukoski, and S. Strausbaugh, "The FeI 3860 circumstellar line towards Beta Pictoris," In preparation.
[83] P. C. Frisch, L. Grodnicki, and D. E. Welty, "The velocity distribution of the nearest interstellar gas," The Astrophysical Journal, vol. 574, no. 2, article 834, 2002.

[84] I. A. Crawford, N. Craig, and B. Y. Welsh, "The velocity structure of the local interstellar medium probed by ultra-high-resolution spectroscopy," Astronomy \& Astrophysics, vol. 317, no. 3, pp. 889897, 1997.

[85] S. Redfield, "Gas absorption detected from the edge-on debris disk surrounding HD 32297," Astrophysical Journal, vol. 656, no. 2, pp. L97-L100, 2007.

[86] J. Zorec and F. Royer, "Rotational velocities of A-type stars: IV. Evolution of rotational velocities," Astronomy \& Astrophysics, vol. 537, article A120, 2012.

[87] E. Paunzen, W. W. Weiss, U. Heiter, and P. North, "A consolidated catalogue of lambda Bootis stars," Astronomy and Astrophysics Supplement Series, vol. 123, no. 1, pp. 93-101, 1997.

[88] E. Solano and E. Paunzen, "Identification of A Bootis stars using IDE spectra II. High resolution data," Astronomy \& Astrophysics, vol. 348, no. 3, pp. 825-830, 1999.

[89] H. A. Abt and N. I. Morrell, "The relation between rotational velocities and spectral peculiarities among A-type stars," Astrophysical Journal, Supplement Series, vol. 99, no. 1, pp. 135-172, 1995.

[90] B. Hauck and C. Jaschek, "A-shell stars in the Geneva system," Astronomy \& Astrophysics, vol. 354, p. 157, 2000.

[91] B. Johnson, C. Lisse, C. Chen et al., "A self-consistent model of the circumstellar debris created by a giant hypervelocity impact in the HD 172555 system," The Astrophysical Journal, vol. 761, no. 1, p. $45,2012$. 

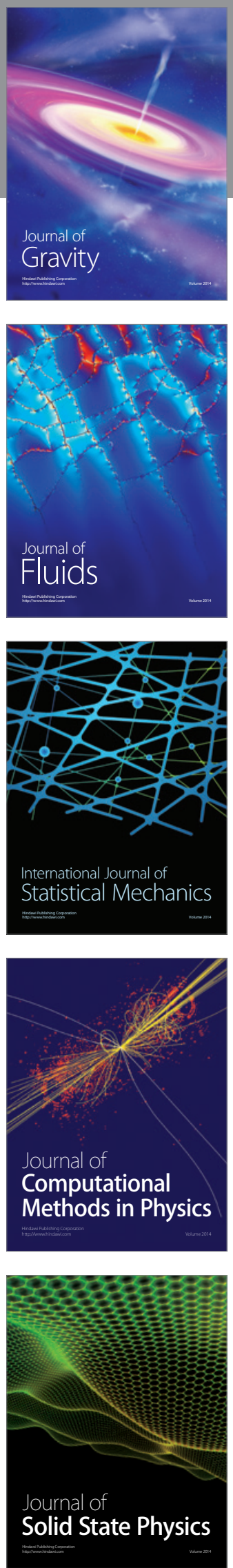

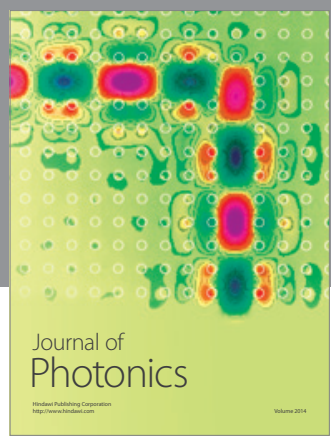

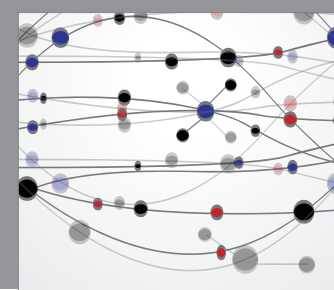

The Scientific World Journal

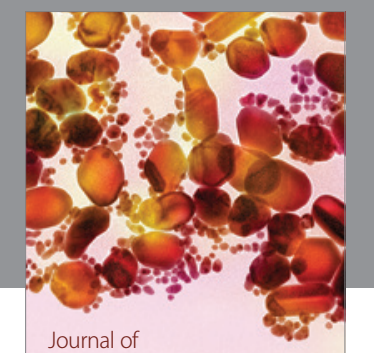

Soft Matter


Submit your manuscripts at

http://www.hindawi.com
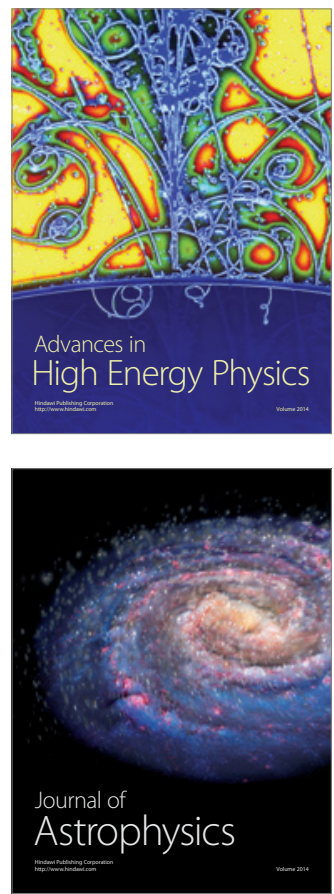
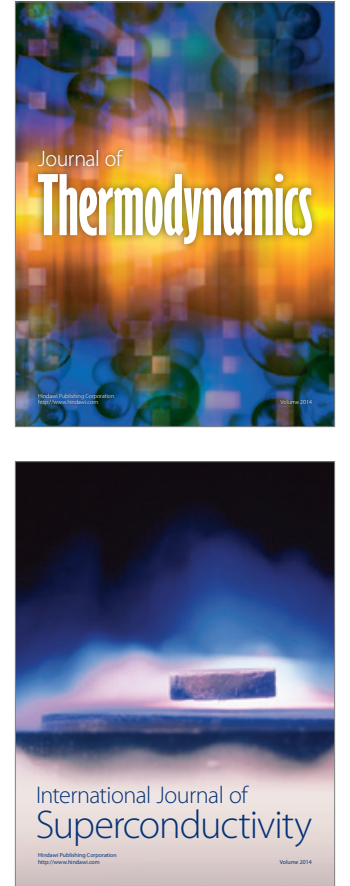
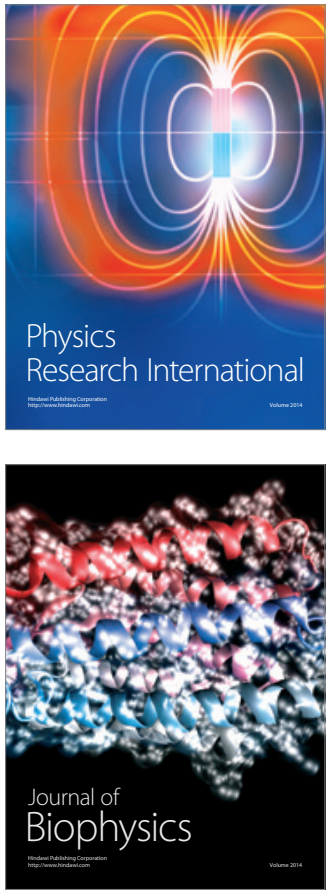
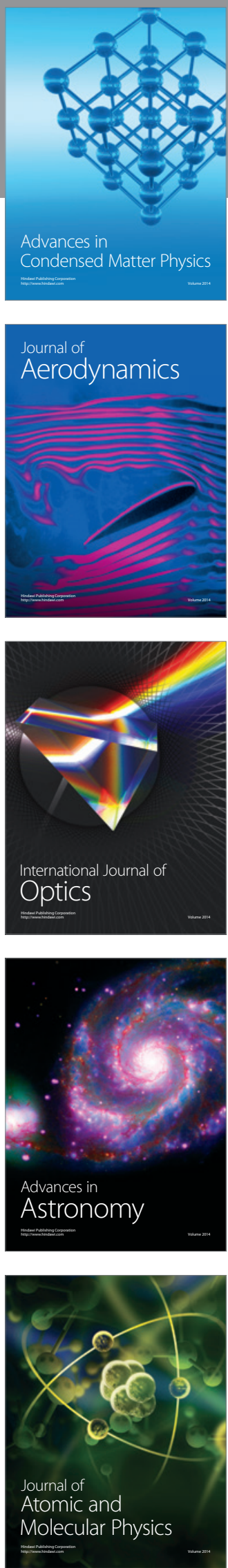